\title{
Inferences on the mantle viscosity structure and the post-overturn evolutionary state of Venus
}

\author{
T. Rolf ${ }^{a, *}$, B. Steinberger ${ }^{b, a}$, U. Sruthi ${ }^{a}$, S. C. Werner ${ }^{a}$ \\ ${ }^{a}$ Centre for Earth Evolution and Dynamics, University of Oslo, 0315 Oslo, Norway \\ ${ }^{b}$ GFZ German Research Centre for Geosciences, Telegrafenberg, 14473 Potsdam, Germany
}

\begin{abstract}
Venus has similar size, density and bulk composition as Earth, but has tectonically evolved clearly differently, and this divergence remains enigmatic. Surface observations such as gravity, topography and surface age constrain Venus' evolution, but interpreting these signals requires understanding of the surface-interior coupling and thus insight into the structure and evolution of the venusian mantle and lithosphere. Here, we investigate how such observables may be generated from interior dynamics using numerical forward models of global mantle convection that consistently link the thermochemical, magmatic and tectonic evolution of Venus. Venus' present surface gravity spectrum and its relation to topography is matched best by our models with a mantle viscosity profile featuring a sublithospheric minimum of $\sim 2 \times 10^{20} \mathrm{Pas}$ and a gradual increase by a factor of $\sim 100$ down to a depth of $\sim 250 \mathrm{~km}$ above the core-mantle boundary. No pronounced viscosity jump around the mantle transition as inferred for Earth is favoured for Venus, which points to a relatively dry venusian upper mantle compared to Earth's as previously suggested. This holds true for both a pure stagnant-lid scenario and in the presence of episodic catastrophic overturns triggered by cumulative crustal growth due to on-going magmatism and volcanism. Overturns strongly perturb the surface gravity spectrum up to $\sim 150 \mathrm{Myr}$ after overturn cessation. Material deeply recycled by the resurfacing event annihilates the developed plume pattern, which needs much longer than those $150 \mathrm{Myr}$ to recover to a state comparable to the pattern suggested by thermal emissivity anomalies observed on Venus. Moreover, overturns limit crustal thicknesses to reasonable values and are more capable than stagnant-lid evolutions in generating mean surface ages $>500 \mathrm{Myr}$. These findings seem to confirm previous suggestions that the episodic regime is more applicable to Venus than a purely stagnant-lid regime. Yet, the relatively long time span required to recycle the entire surface $(\sim 150-200 \mathrm{Myr})$ and the presently on-going volcanic resurfacing predicted by our models complicate the formation of a uniform surface age as indicated by Venus' crater population and may also suggest that the latest
\end{abstract}

\footnotetext{
${ }^{*}$ Corresponding author

Email address: tobias.rolf@geo.uio.no (T. Rolf)
} 
overturn has ceased longer ago than indicated by Venus' present mean surface age.

Keywords: Venus, Mantle evolution, Gravity Spectrum, Viscosity structure

\section{Introduction}

Venus is regularly called Earth's "sister planet", but clearly the evolutions of both planets have diverged at some point. This is ultimately expressed by the current operation of plate tectonics on Earth, but not on Venus. The absence of continuous plate tectonic recycling on Venus may explain several first-order observations made about our closest planetary neighbour, such as the dearth of an internally generated magnetic field and the presence of a dense, dry $\mathrm{CO}_{2}$-rich atmosphere (e.g. Driscoll and Bercovici, 2013; Gillmann and Tackley, 2014). The reason for the tectonic discrepancy of the two bodies has remained an enigma in planetary dynamics for many years. Possible explanations range from Venus' much higher surface temperature, which enhances healing of tectonic damage and thus complicates the development of tectonic plates (e.g. Bercovici and Ricard, 2014), to a lower water content of the venusian interior, to bi-stable planetary evolutions, in which multiple tectonic regimes would be equally feasible over a range of plausible conditions (e.g. Weller et al., 2015). In the latter case, stochastic perturbations, for instance early asteroidal impacts (see e.g. O'Neill et al., 2017) may be sufficient to change the tectonic evolution scenario.

Most suggestions demonstrate the importance of the coupling between surface and interior. For instance, melting and outgassing of Venus' interior may have caused its dehydration and the development of thick atmosphere as well as high surface temperature, which then prevented sufficient long-term damage of Venus' surface rocks to establish an Earth-like crustal recycling mechanism. On Earth, the surface plates are mostly an expression of large-scale convection in the deep interior (Bercovici, 2003), but Venus' surface lacks continuously mobile and subducting plates. As such, surface-interior coupling may function differently. Yet, the details of such a presumed coupling remain insufficiently understood.

Understanding these issues requires advanced insights into (1) Venus' present interior state in comparison to Earth's and (2) the planet's evolutionary path given the absence of clear features related to plate recycling. Concerning (1), direct observations of Venus' interior are essentially not available due to the lack of seismic measurements, so that inferences on the deep interior can only be drawn indirectly, for example by interpreting surface signals such as gravity and topography. At least at long-wavelength, these signals are linked to the structure and dynamics of the sublithospheric mantle (Pauer et al., 2006; Steinberger et al., 2010), but gravity interpretation is usually non-unique (see e.g. Wieczorek, 2007) and has to be embedded into (2) a consistent context of Venus' evolution.

Currently, Venus may be in the stagnant-lid mode of convection (see e.g. 
Solomatov, 1995) in which the shallow lithosphere can uplift and subside (dynamic topography), but is not substantially weakened and structured by tectonic forces arising from deep mantle flow compared to Earth. Subduction-like processes on Venus have been proposed though (Schubert and Sandwell, 1995), perhaps triggered by mantle plumes (Davaille et al., 2017), so that Venus may be in a transitional regime between an Earth-like continuously mobilised lithosphere and a more conventional stagnant-lid, such as contemporary Mars or Mercury.

This transitional regime may be characterised by episodic mobile-lid behaviour during earlier phases of Venus' evolution. The observed, roughly random, distribution of impact craters on Venus (Herrick, 1994) in fact suggests a rather young $\left(750_{-450}^{+250} \mathrm{Myr}\right.$, McKinnon et al., 1997) and essentially uniform surface age, which favours a global event of surface mobilisation and tectonic resurfacing around that time (e.g. Romeo and Turcotte, 2010). Nonetheless, crater statistics have large uncertainties and the comparably small number of craters on Venus also strongly limits the length scales over which inferences can be made, so that the possibility of equilibrium resurfacing such as by volcanism, cannot be rejected per se (e.g. Bjonnes et al., 2012). A hybrid mode in which episodic tectonic recycling cools the interior and subsequently reduces the intensity of the (still on-going) volcanic resurfacing may provide the most feasible scenario. We come back to this in section 3.3.

The uncertainties about the style and evolution of Venus' convective regime complicate the interpretation of present-day observables. In particular, it is not sufficiently understood how such observables may depend on Venus' convective regime; specifically, how they would respond to episodes of surface mobilisation and tectonic recycling. If the aftermath of a global overturn lasts short compared to Venus' characteristic surface age, then present Venus may be representative of the stagnant-lid mode. But if remnants of the latest overturn episode can persist sufficiently long in Venus' interior, this could affect the present flow pattern and structure in the mantle, which could be reflected in present surface observables such as topography and gravity. Yet, the aftermath of a global overturn and its thermochemical and magmatic consequences remain insufficiently understood.

To gain deeper insight into these aspects, it is necessary to decipher the relation between deep mantle structure and dynamics as well as surface observables in a consistent evolutionary framework. Here, we use a numerical model of Venus' interior that links together the thermochemical, magmatic and tectonic evolution of the planet. We employ this framework in order to (1) constrain a likely present-day structure of Venus' mantle focussing on mantle viscosity and (2) to make inferences on the evolutionary state on Venus in relation to the proposed global resurfacing events.

\section{Methodology}

\subsection{Numerical model}

We compute the thermochemical evolution of Venus' interior using the mantle convection code StagYY (Tackley, 2008). Our setup is similar to the one 
used in Armann and Tackley (2012), where additional model details can be found. A major difference is that we use a 3D spherical rather than 2D annulus geometry. On the other hand our incorporation of mineral physics is simpler in the sense that we assume the venusian mantle to be incompressible. Specifically, we employ the extended Boussinesq approximation in which the terms for viscous dissipation and adiabatic heating are included in the energy equation, which makes it more consistent with regards to latent heat effects, such as those related to the presence of phase transitions (see Christensen and Yuen, 1985). Consequently, our models include an adiabatic temperature increase across the entire mantle of $\sim 850 \mathrm{~K}$.

Since our model assumes that the mantle is an incompressible fluid, density cannot explicitly depend on depth in the extended Boussinesq limit, but other thermodynamic parameters could. This concerns mainly thermal expansivity, but also thermal conductivity and the gravitational acceleration. We do not consider variations of these parameters due to temperature and/or pressure variations (see discussion in section 4) as this strategy allows us to compare our results more directly to those of Huang et al. (2013). These authors also employed the extended Boussinesq limit without depth variation of thermodynamical parameters and gravity; but their treatment neglected overturn scenarios like the ones considered here. In contrast, Armann and Tackley (2012) employed a 2 D compressible, anelastic model including radial variation of thermodynamical parameters (not gravity) and also considered overturn episodes. Yet, our study discusses several diagnostics with inherent 3D nature (see section 2.3), such as the planetary gravity spectrum, the spatial pattern of mantle plumes and the modes of crustal recycling that impact the surface age distribution. As a consequence, we preferred using 3D models for the sake of better comparability to observations; the cost of this is a somewhat reduced complexity of our physical model compared to the one of Armann and Tackley (2012).

The model domain is a 3D spherical shell with core radius $R_{C}$ and surface radius $R_{S}$, which has free-slip mechanical boundary conditions at both boundaries. The shell is discretised on a YinYang grid whose two grid blocks have a resolution of $64 \times 192 \times 64$ cells each. The radial grid spacing is refined to $\sim 20-25 \mathrm{~km}$ close to the surface, near the phase changes in the transition zone and at the core-mantle boundary. The shell is cooled from above $\left(T=T_{S}\right)$ and heated from below $\left(T=T_{C}(t)\right)$ as well as from within using a generic bulk heating rate of $H(t)=H_{P} \exp (t / \tau \ln 2)$.

In most model cases, we chose the present-day value $H_{P}=H(t=0 \mathrm{Ga})=$ $5 \times 10^{-12} \mathrm{~W} \mathrm{~kg}^{-1}$, but in two cases we used reduced values to test the effect of this parameter. Our nominal value of $H_{P}$ is comparable to, but at the lower end of the range inferred for present-day Earth (see e.g. Turcotte and Schubert, 2002). We employed a decay constant of $\tau=3 \mathrm{Gyr}$, such that the internal heating rate at model initiation $\left(t=t_{0}=4.4 \mathrm{Ga}\right)$ is about $2.8 \times$ higher than at present day. Radiogenic decay is thus somewhat less pronounced than during Earth's, and Venus' history, implying that our models may feature somewhat less radiogenic heating during the earlier stages of evolution. While this is to be improved in future models, we made this choice to limit the vigour of convection 
and the degree of partial melting (see below), which require extra treatment and would significantly increase computational costs. Our present study is mostly concerned about present-day observables and Venus' late-stage history, but we note that inheritance from earlier periods could play a role in some aspects.

Besides its overall amount, internal heat is generated uniformly across the mantle except in two cases where it is more enriched by a factor $\Delta H$ in the basaltic material that typically represents the crust (see below). Surface temperature is constant through time (cf. Gillmann and Tackley, 2014), but coremantle boundary $(\mathrm{CMB})$ temperature $T_{C}$ evolves in time from its initial value $T_{c 0}$ in response to the heat flow $Q_{C}$ across the CMB as $d T_{C} / d t=-Q_{C} / M_{C} c_{p, C}$, where $M_{C}$ is the core mass and $c_{p, C}$ is the core's specific heat capacity. This simple core model does not consider the possibility of internal heat generation in the core or other complexities like inner core crystallisation. However, our knowledge of Venus' core is very fragmentary so that a more sophisticated approach would lack sufficient ground truth.

\subsubsection{Composition and mineralogy}

Material composition is tracked by $1.44 \times 10^{8}$ tracer particles using the tracerratio method (Tackley and King, 2003). This corresponds to an average of $\sim 90$ tracers per grid cell. The tracers are used to map composition into a continuous field $(0 \leq C \leq 1)$ that represents a mixture of two end-member components: basalt $(C=1)$ and harzburgite $(C=0)$. Basalt is assumed to consist of $100 \%$ pyroxene-garnet, while harzburgite is composed of $25 \%$ pyroxene-garnet and $75 \%$ olivine (see Xie and Tackley, 2004).

Initially, the described mixture is homogeneous in the entire model (see section 2.2), but it evolves with time depending on the thermal and magmatic history of the material: if the temperature of a patch exceeds its solidus, its basaltic components can partially melt and composition changes. The solidus is here given by a fit to experimental data on Earth's mantle rocks (see Xie and Tackley, 2004). Upon melting, latent heat is consumed. In the upper mantle, melt is assumed to be buoyant and to rise quickly. This process is simplified here by an immediate extraction of melt from the mantle; the extracted material is emplaced at the surface as basaltic crust. The residue is depleted in basalt and becomes more difficult to melt: the solidus increases linearly with decreasing basalt content (by up to $150 \mathrm{~K}$ when the basalt fraction approaches $0 \%$ ). Generally, melt extraction is limited to the upper mantle here, that is above $730 \mathrm{~km}$.

Some major phase transitions are included in our model, as they may be important for generating mantle flow patterns consistent with Venus' geoid (Huang et al., 2013). These transitions happen at somewhat greater depth than on Earth due to the lower venusian gravity. The olivine and the pyroxene-garnet system have distinct phase transitions (see Xie and Tackley, 2004; Armann and Tackley, 2012, for details). Specifically, olivine converts into magnesiowüstite at $d=450 \mathrm{~km}$ and further into perovskite at $d=730 \mathrm{~km}$ depth. The pyroxenegarnet system considers three transitions at depths of 65,440 , and $800 \mathrm{~km}$ the first of which describes the transition from basalt to eclogite. The assumed prop- 


\begin{tabular}{l|l|l} 
Symbol & Definition & Value \\
\hline$R_{S}$ & Planetary mean radius & $6052 \mathrm{~km}$ \\
$R_{C}$ & Core radius & $3186 \mathrm{~km}$ \\
$D_{0}$ & Mantle thickness & $2866 \mathrm{~km}$ \\
$g_{0}$ & Gravitational acceleration & $8.87 \mathrm{~m} \mathrm{~s}^{-2}$ \\
$T_{S}$ & Surface temperature & $740 \mathrm{~K}$ \\
$T_{C 0}$ & Initial CMB temperature & $3850 \mathrm{~K}$ \\
$\Delta T$ & Superadiabatic temperature drop & $2300 \mathrm{~K}$ \\
$H_{P}$ & Present-day bulk internal heating rate & $5 \times 10^{-12} \mathrm{~W} \mathrm{~kg}^{-1}$ \\
$\tau$ & Radiogenic decay constant & $3.0 \times 10^{9} \mathrm{yr}^{-3}$ \\
$\rho_{0}$ & Mantle density & $3378 \mathrm{~kg} \mathrm{~m}^{-3}$ \\
$k_{0}$ & Mantle thermal conductivity & $4 \mathrm{~W} \mathrm{~m}-1 \mathrm{~K}^{-1}$ \\
$c_{p_{0}}$ & Mantle specific heat capacity & $1250 \mathrm{Jgg}^{-1} \mathrm{~K}^{-1}$ \\
$c_{p, C}$ & Core specific heat capacity & $800 \mathrm{Jg}^{-1} \mathrm{~K}^{-1}$ \\
$\alpha_{0}$ & Mantle thermal expansivity & $2 \times 10^{-5} \mathrm{~K}^{-1}$ \\
$E_{A}$ & Activation energy & $2 \times 10^{5} \mathrm{~J} \mathrm{~mol}^{-1}$ \\
$\mu$ & Friction coefficient & 0.5 \\
$L_{m}$ & Latent heat of melting & $6 \times 10^{5} \mathrm{~J} \mathrm{~kg}^{-1}$ \\
$\Delta \rho_{S}$ & Surface density jump & $3318 \mathrm{~kg} \mathrm{~m}^{-3}$ \\
$\Delta \rho_{C}$ & CMB density jump & $4357 \mathrm{~kg} \mathrm{~m}^{-3}$ \\
$\Delta \rho_{o l_{450 / 730}}$ & Density jumps (ol) & $250 / 150 \mathrm{~kg} \mathrm{~m}^{-3}$ \\
$\Delta \rho_{p x_{65 / 440 / 800}}$ & Density jumps (px) & $250 / 150 / 150 \mathrm{~kg} \mathrm{~m}^{-3}$ \\
$\gamma_{o l_{450 / 730}}$ & Clapeyron slopes (ol) & $2 /-2 \mathrm{MPa} \mathrm{K}^{-1}$ \\
$\gamma_{p x_{65 / 44 / 800}}$ & Clapeyron slopes (px) & $0 / 1 / 1 \mathrm{MPa} \mathrm{K}^{-1}$ \\
& & \\
& &
\end{tabular}

Table 1: Symbols, definitions and reference values used in this study. The last four rows contain multiple values that describe the respective values for the different phase transitions: either 2 in the olivine (ol) system or 3 in the pyroxene-garnet (px) system. The numeric subscripts in these variable names denote the depth of the phase transitions in kilometres.

erties of all phase transitions, such as Clapeyron slopes and density increases, are kept fixed here and are summarised in Table 1.

\subsubsection{Viscosity calculation}

In this section, we describe how our model computes an effective material viscosity. In summary, effective viscosity $\eta$ is computed as the harmonic average of two contributions of which the first one, $\eta_{1}$, is described by an Arrhenius law

$$
\eta_{1}=A \eta_{p} \exp \left(\frac{E_{A}+p V_{A}}{R_{g} T}\right),
$$

where $A=A\left(\eta_{0}\right)$ is a pre-factor that forces $\eta_{1}$ to be equal to the reference viscosity $\eta_{0}$ at temperature $T=1613 \mathrm{~K}$ and pressure $p=0 \mathrm{~Pa}$. The term $\eta_{p}$ describes the phase dependence. For simplicity, we only consider a viscosity increase across the transition to perovskite, that is the lower mantle phase in which $\eta_{p}=\Delta \eta_{p}$, so that $\eta_{p}=1$ elsewhere. By convention, $R_{g}$ is 
the gas constant and $V_{A}$ the activation volume, which depends on pressure as $V_{A}(p)=V_{A 0} \exp \left(-p / p^{*}\right)$ (Tackley et al., 2013). Here, we use $p^{*}=400 \mathrm{GPa}$ to account for the reduction of activation volume in the lowermost mantle and $V_{A 0}$ is the activation volume at $p=0 \mathrm{~Pa}$. We assign the activation energy $E_{A}$ a moderate value of $200 \mathrm{~kJ} \mathrm{~mol}^{-1}$ as a compromise between realism and numerical feasibility. However, the chosen value is large enough to allow for strong thermal viscosity variation and thus for the formation of a stagnant lid on top of the convecting mantle (see Solomatov, 1995). For simplicity, viscosity does not explicitly depend on composition and the rheological parameters except $\eta_{p}$ are independent of phase (see Table 1). For numerical reasons, the range over which viscosity can vary is limited to the interval $\left[10^{18}, 10^{25}\right] \mathrm{Pas}$.

In the stagnant-lid set of calculations (described below), effective viscosity is completely determined by $\eta_{1}$. In some cases we employ a viscoplastic rheology to allow the lithosphere to fail plastically when the convective stresses reach the yield stress $\sigma_{Y}$. The yield stress is depth-dependent based on Byerlee's law using a friction coefficient of $\mu=0.5$ (and a cohesion of $0 \mathrm{~Pa}$ ), but is bounded by a maximum value $\sigma_{0}$, thus,

$$
\sigma_{Y}=\min \left(\mu p, \sigma_{0}\right)
$$

In case of plastic yielding, the viscosity is reduced to $\eta_{2}=\sigma_{Y} / 2 \dot{\epsilon}$, where $\dot{\epsilon}$ is the 2nd invariant of the strain rate tensor, and the effective viscosity is then given by $\eta=\left(1 / \eta_{1}+1 / \eta_{2}\right)^{-1}$. This method has turned out to be a viable parametrisation to generate large-scale lithospheric overturn events that may have occurred during Venus' evolution (e.g. Moresi and Solomatov, 1998; Armann and Tackley, 2012).

\subsection{Computed evolutions}

We compute Venus' mantle evolution from $t_{0}=4.4 \mathrm{Ga}$ until present-day as described above. We chose this rather late initiation time $t_{0}$, because we assume a solid-state interior in our model that does not properly capture the dynamics of a largely molten mantle during the initial stages of planetary thermal evolution. In all cases, we use the same initial condition in which temperature increases from its fixed surface value across an $80 \mathrm{~km}$ thick boundary layer to an internal temperature of $\sim 1900 \mathrm{~K}$, which assumes that mantle temperature was higher early in planetary evolution than at present (see e.g. Herzberg et al., 2010). Below the boundary layer, temperature increases adiabatically until the bottom boundary layer is reached. At this point, temperature increases strongly to the initial CMB value. The entire mantle is initialized with a bulk composition of $C=0.2$, that is $80 \%$ harzburgite and $20 \%$ basalt and consequently a mix of $60 \%$ olivine and $40 \%$ pyroxene (see section 2.1.1).

We compute two sets of evolutions. In the first set, the stagnant-lid (S)family, we compute 10 evolutions in which plastic failure of the lithosphere is inhibited. In these cases, we focus on the role of the rheological parameters $\eta_{0}, V_{A 0}$, and $\Delta \eta_{p}$ in the evolution of venusian mantle dynamics and its surface expressions. After defining a preferred case, we use the rheological parameters of 


\begin{tabular}{l|c|c|c|c|c|c} 
Case & $\boldsymbol{\eta}_{\mathbf{0}}$ & $\boldsymbol{V}_{\boldsymbol{A} \mathbf{0}}$ & $\boldsymbol{\Delta} \boldsymbol{\eta}_{\boldsymbol{p}}$ & $\boldsymbol{\sigma}_{\mathbf{0}}$ & $\boldsymbol{H}_{\boldsymbol{P}}$ & $\boldsymbol{\Delta} \boldsymbol{H}$ \\
\hline S1 & 0.3 & 3.5 & 1 & - & 5 & 1 \\
S2 & 1 & 3.5 & 1 & - & 5 & 1 \\
S2a & 1 & 3.5 & 1 & - & 4 & 1 \\
S2b & 1 & 3.5 & 1 & - & 5 & 10 \\
S3 & 3 & 3.5 & 1 & - & 5 & 1 \\
S4 & 10 & 3.5 & 1 & - & 5 & 1 \\
S5 & 1 & 2.5 & 1 & - & 5 & 1 \\
S6 & 1 & 4.5 & 1 & - & 5 & 1 \\
S7 & 1 & 3.5 & 5 & - & 5 & 1 \\
S8 & 1 & 3.5 & 20 & - & 5 & 1 \\
\hline E30 & 1 & 3.5 & 1 & 30 & 5 & 1 \\
E50 & 1 & 3.5 & 1 & 50 & 5 & 1 \\
E50a & 1 & 3.5 & 1 & 50 & 4 & 1 \\
E50b & 1 & 3.5 & 1 & 50 & 5 & 10 \\
E55 & 1 & 3.5 & 1 & 55 & 5 & 1 \\
E60 & 1 & 3.5 & 1 & 60 & 5 & 1 \\
E70 & 1 & 3.5 & 1 & 70 & 5 & 1
\end{tabular}

Table 2: List of performed calculations and their characterising parameters, $\eta_{0}, V_{A 0}, \Delta \eta_{p}$, $\sigma_{0}, H_{P}$, and $\Delta H$. Values of $\eta_{0}$ are scaled with a value of $10^{21} \mathrm{Pas}$, values of $V_{A 0}$ are given in $10^{-6} \mathrm{~m}^{3} \mathrm{~mol}^{-1}$ and those for $H_{P}$ in $10^{-12} \mathrm{~W} \mathrm{~kg}^{-1}$. Stagnant cases (S) do not consider a yield stress, while episodic cases (E) feature finite values, given in MPa. In the latter set, the numeric part of the case name denotes the value of $\sigma_{0}$ in $\mathrm{MPa}$.

this case to compute a second set of 7 calculations in which episodic lithospheric failure is allowed for (dubbed the E-family). In this set, we mostly focus on variation of the yield stress, which determines how easily the lithosphere can fail. An overview of the computed cases and their characterising parameters is given in Table 2.

\subsection{Diagnostics}

For all cases, we compute a number of diagnostics and the resulting values are summarised in Table 3. In particular, we compute the average heat flux across the surface $\left(q_{S}\right)$ and CMB $\left(q_{C}\right)$ at present-day, which are given by Fourier's law $\overline{q_{S, C}}=-k_{S, C} \overline{\partial T / \partial r}$. In addition, we compute the number of hot mantle plumes (described in section 3.1.3) and crustal thickness (see section 3.3.1). Primarily though, we compute the surface geoid under self-gravitation following the approach described in Zhong et al. (2008), which is based on Zhang and Christensen (1993). We describe this approach only conceptually here, for a more detailed description including equations we refer to these papers. We first use the thermochemical density heterogeneities arising from integrating the evolution equations (Stokes flow) forward in time and convert them into spectral space $\left(\delta \rho_{l m}\right)$, where $l$ and $m$ are degree and order of the spherical harmonic, respectively. Such density anomalies drive the large-scale convective flow and thus lead to deflection of the surface and CMB (Hager et al., 1985); 
these define the dynamic topography.

With the free-slip boundary conditions in our model, these deflections are estimated from the respective normal stress acting on these boundaries as well as the density jumps $\Delta \rho_{s}$ and $\Delta \rho_{c}$ across these boundaries, and are also converted into spectral space $\left(\delta s_{l m}\right.$ and $\left.\delta c_{l m}\right)$. With $\delta \rho_{l m}, \delta s_{l m}$ and $\delta c_{l m}$ the gravitational potential at the surface $\left(\Phi_{l m}\right)$ and $\mathrm{CMB}\left(\Psi_{l m}\right)$ can in principle be estimated, but both $\delta s_{l m}$ and $\delta c_{l m}$ depend in turn on these potentials. In the spectral domain it is possible though to couple the expressions and solve for $\Phi_{l m}, \Psi_{l m}, \delta s_{l m}$ and $\delta c_{l m}$ simultaneously for a given combination of $l$ and $m$ (see e.g. Appendix $\mathrm{A}$ in Zhong et al., 2008). The same method has already been used in StagYY models of Venus' interior by Armann and Tackley (2012), but their 2D spherical annulus models could only capture sectoral harmonics (i.e., $l=m$ ), so that not the entire spectrum was included in their analysis.

Once $\Phi_{l m}$ has been computed in the spectral domain (we do not further discuss $\Psi_{l m}$ here), it is straightforward to synthesise it into its power spectrum $P_{g g}$. Following Steinberger and Holme (2002), we define the dimensionless power spectrum as

$$
P_{g g}(l)=(l+1)\left(g_{l 0}^{2}+\sum_{m=1}^{l} g_{l m}^{2}+h_{l m}^{2}\right),
$$

where $g_{l m}$ and $h_{l m}$ are the fully normalised spherical harmonic expansion coefficients of the gravitational potential. Here, we only consider the degree range of spherical harmonics as $2 \leq l \leq 16=l_{\max }(m \leq l)$. In this range, $P_{g g}$ is likely predominantly generated by deep (mantle) rather than shallow (crustal) sources and can thus be used to make inferences on the structure of the deep interior at this range of spatial wavelengths (Steinberger et al., 2010). In an analogous way, we can define the power spectrum of surface topography $P_{t t}$ and the cross-power spectrum $P_{g t}$, which we use to build the degree correlation $C_{g t}(l)=P_{g t} / \sqrt{P_{g g} P_{t t}}$ and the spectral ratio $R_{g t}(l)=\sqrt{P_{g g} / P_{t t}}$ of gravity and topography.

Our approach assumes a purely viscous body and does not involve elastic effects within the lithosphere (e.g. Turcotte et al., 1981). These change the displacement of the surface (i.e. topography) upon loading and consequently the gravity signal, in particular when lithospheric thickness is large. For internal loads that are most relevant for the present study, however, Steinberger et al. (2015) suggest that the resulting reduction in topography may not be as large as originally proposed by Turcotte et al. (1981). The elastic contributions could be derived after estimating the elastic properties such as the (time-dependent) elastic thickness of the lithosphere. But we do not consider this here for simplicity and for the sake of comparability to the study of Steinberger et al. (2010), who suggested that a purely viscous rather than a viscoelastic model can explain the long-wavelength gravity and topography on Venus.

For comparison to observational data from Venus we use gravity model SHGJ180U.A01 (available online at http://pds-geosciences.wustl.edu/mgn/mgn$v$-rss-5-gravity-l2-v1/mg_5201/gravity/). The topography we use here is obtained from Venus' shape as given by Wieczorek (2007); these data are avail- 
able online at https://markwieczorek.github.io/web/spherical-harmonic-modelstopography/spherical-harmonic-shape-models.html. We focus on spectral characteristics here, because our generic numerical model cannot be expected to reproduce the actual observed venusian gravity and topography patterns in real space. However, the obtained solutions for $\Phi_{l m}$ could in principle be transformed into real space.

To quantify how well our model predictions match the present-day observations, we define the misfit measures $\delta_{G}, \delta_{T}, \delta_{C}$ and $\delta_{R}$. For example, $\delta_{G}$ is given as $\delta_{G}=\left[1 /\left(l_{c}-1\right) \cdot \sum_{i=2}^{l_{c}}\left(\left(P_{g g, i}-P_{g g, i}^{*}\right) / P_{g g, i}^{*}\right)^{2}\right]^{1 / 2}$, where $P_{g g}^{*}$ is the gravity power for present-day Venus. $\delta_{G}$ may thus be interpreted as the average relative misfit between predicted and observed power spectrum per spherical harmonic degree. A value of $\delta_{G}=0$ implies a perfectly matching model, while a value of 1 means that the misfit has the same amplitude as the respective observational value. $\delta_{T}, \delta_{C}$, and $\delta_{R}$ are defined accordingly using either the topography power spectrum $P_{t t}$, the degree correlation $C_{g t}$ or the geoid-topography ratio $R_{g t}$ instead of $P_{g g}$. We chose $l_{c}=10$ because we are primarily interested in the misfit at the longest wavelengths, which are more sensitive of the structure of the deeper interior. Finally, we note that we will subsequently plot the square root of $P_{g g}$ multiplied with the factor $G M$ (where $G$ is the gravitational constant and $M$ is the planetary mass), simply because this measure has the intuitive unit of meters.

\section{Results}

\subsection{Stagnant-lid models}

\subsubsection{Thermal and magmatic evolution}

We first present a reference model (case S2) to demonstrate some general features of the computed evolutions in the stagnant-lid scenario. Largely, the thermal evolution is characterised by an initial phase of heating of the mantle during which the radiogenic heat production dominates over the entire (bulk) mantle (Figure 1a). A peak in bulk mantle temperature is reached at $\sim 3 \mathrm{Ga}$ in this case, after which the effects of secular cooling and surface heat loss overcome the heating of the mantle, and mantle temperature starts to drop with an almost constant rate of $\sim 25-30 \mathrm{~K} \mathrm{Gyr}^{-1}$. This rather slow rate of cooling is due to the absence of surface recycling via subduction of lithospheric plates back into the mantle. Aside from secular cooling that arises from the slow demise of radiogenic heating, heat is lost by conduction through the lithosphere and by the extraction of melt. In total, this accounts for an average surface heat flux of $\sim 22 \mathrm{~mW} \mathrm{~m}^{-2}$ at present-day, a factor $4-5$ smaller than the average terrestrial heat flux at present-day.

Except during the first billion years of evolution, this value has not been much different for earlier times, but a general tendency towards a gently increasing surface heat flux $\left(q_{S}\right)$ by a few $\mathrm{mW} \mathrm{m}^{-2}$ in the second half of the evolution is apparent (Figure 1b). We attribute this to the slow decrease in thermal boundary layer thickness with time (Figure 1d), which itself is linked 


\begin{tabular}{l|c|c|c|c|c|c|c|c|c} 
Case & $\overline{\boldsymbol{q}_{\boldsymbol{S}}}$ & $\overline{\boldsymbol{q}_{\boldsymbol{C}}}$ & $\boldsymbol{M}_{\boldsymbol{e}}^{\text {tot }}$ & $\boldsymbol{\delta}_{\boldsymbol{G}}$ & $\boldsymbol{\delta}_{\boldsymbol{T}}$ & $\boldsymbol{\delta}_{\boldsymbol{C}}$ & $\boldsymbol{\delta}_{\boldsymbol{R}}$ & $\overline{\boldsymbol{N}_{\boldsymbol{p l}}}$ & $\overline{\boldsymbol{d}_{\boldsymbol{c} \boldsymbol{r}}}$ \\
\hline S1 & 25.5 & 20.8 & 0.41 & 0.71 & 0.47 & 0.51 & 0.18 & $8.8 \pm 0.6$ & $109 \pm 11$ \\
S2 & 21.9 & 18.7 & 0.34 & 0.52 & 0.40 & 0.48 & 0.25 & $9.4 \pm 0.5$ & $136 \pm 15$ \\
S2a & 23.1 & 19.2 & 0.25 & 1.31 & 0.74 & 0.49 & 0.28 & $11.5 \pm 2.1$ & $131 \pm 13$ \\
S2b & 27.1 & 19.4 & 0.31 & 0.69 & 0.34 & 0.49 & 0.29 & $8.6 \pm 0.6$ & $123 \pm 14$ \\
S3 & 18.9 & 15.9 & 0.30 & 1.85 & 1.52 & 0.48 & 0.23 & $16.0 \pm 1.3$ & $168 \pm 23$ \\
S4 & 15.1 & 13.6 & 0.27 & 12.2 & 7.36 & 0.38 & 0.51 & $27.2 \pm 2.8$ & $198 \pm 26$ \\
S5 & 22.7 & 16.9 & 0.40 & 0.73 & 0.59 & 0.57 & 1.25 & $7.6 \pm 1.5$ & $131 \pm 98$ \\
S6 & 21.8 & 15.5 & 0.29 & 2.40 & 1.45 & 0.49 & 0.44 & $14.6 \pm 1.0$ & $139 \pm 22$ \\
S7 & 22.2 & 18.4 & 0.33 & 1.02 & 0.53 & 0.50 & 0.36 & $12.6 \pm 0.5$ & $135 \pm 17$ \\
S8 & 22.6 & 17.3 & 0.31 & 2.68 & 0.98 & 0.73 & 1.24 & $13.6 \pm 0.6$ & $132 \pm 18$ \\
\hline E30 & 42.6 & 32.6 & 0.34 & 1.44 & 0.41 & 0.78 & 0.61 & $-{ }^{*}$ & $21 \pm 7$ \\
E50 & 26.0 & 15.3 & 0.30 & 0.26 & 0.30 & 0.33 & 0.21 & $-{ }^{*}$ & $62 \pm 24$ \\
E50a & 25.2 & 15.5 & 0.22 & 0.34 & 0.35 & 0.43 & 0.31 & $-{ }^{*}$ & $58 \pm 22$ \\
E50b & 27.5 & 14.6 & 0.30 & 0.49 & 0.29 & 0.22 & 0.34 & $-{ }^{*}$ & $44 \pm 19$ \\
E55 & 27.5 & 15.8 & 0.32 & 0.55 & 0.49 & 0.64 & 0.24 & $-{ }^{*}$ & $48 \pm 18$ \\
E60 & 29.8 & 31.2 & 0.33 & 0.48 & 0.88 & 0.25 & 0.21 & $-{ }^{*}$ & $67 \pm 50$ \\
E70 & 23.3 & 16.0 & 0.30 & 0.39 & 0.39 & 0.44 & 0.18 & $-{ }^{*}$ & $114 \pm 25$
\end{tabular}

Table 3: Output diagnostics: mean surface heat flux $\overline{q_{S}}$, mean CMB heat flux $\overline{q_{C}}$, total cumulative mass of erupted material $M_{e}^{\text {tot }}$ (normalised by the total mantle mass), average deviations from the observed gravity power spectrum $\delta_{G}$, topography spectrum $\delta_{T}$, gravitytopography correlation $\delta_{C}$, and from the observed gravity-topography ratio $\delta_{R}$, number of detected plumes $\overline{N_{p l}}$ and mean crustal thickness $\overline{d_{c r}}$. If given, \pm -symbols indicate one standard deviation. All values are for $t=0 \mathrm{Ga}$. Heat fluxes are given in $\mathrm{mW} \mathrm{m}^{-2}$ and crustal thicknesses in $\mathrm{km}$, respectively. For $N_{p l}$, we provide a mean value and standard deviation from 100 different detection thresholds $\xi_{1,2} . \quad\left({ }^{*} \bar{N}_{p l}\right.$ is not listed here for episodic cases, because it becomes more time-dependent and sensitive to the detection thresholds.

to a slow decrease in melt production and extraction (Figure 1c) and thus in crustal thickness (see section 3.1.3). On the other hand, the heat flux from the core into the mantle decreases by a factor of $\sim 3$ from 4 Ga until present-day (and by a factor of $\sim 2$ from $3 \mathrm{Ga}$, respectively). This is because in the modelled stagnant-lid scenario the mantle does not cool quickly enough, so that internal mantle temperature and CMB temperature slowly adjust (Figure 1d), which effectively reduces the heat flow across this boundary.

Some part of the cooling of the mantle is always due to the extraction of hot magma and its emplacement at the surface where it cools quickly (Armann and Tackley, 2012) (assuming that the surface temperature is not substantially higher than for present-day Venus). The magnitude of this contribution depends on the temperature within the upper mantle and thus follows a similar decreasing trend as mantle temperature. The models show that melting and magmatic eruptions are still ongoing at present-day (Figure 1c) and thus contribute to cooling the interior and resurfacing. As a consequence, the entire mantle below the crust is depleted in basalt compared to the initial bulk composition (Figure 1e). Melting occurs only in the upper mantle, but mantle flow homogenises composition fairly efficiently, so that no clearly basalt enriched regions seem to 

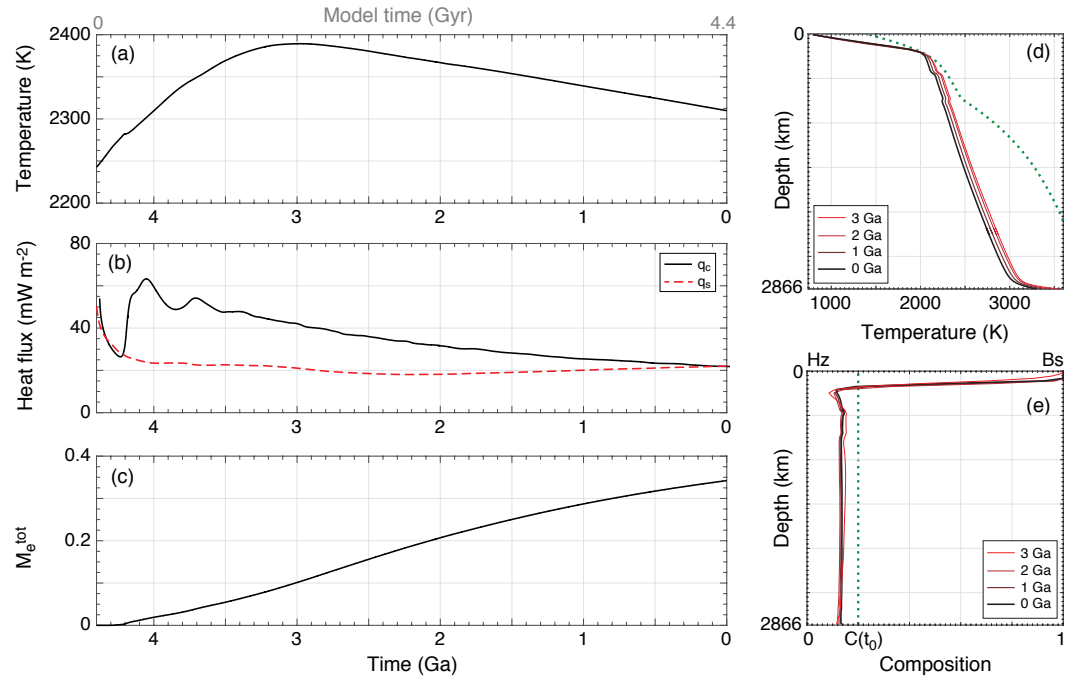

Figure 1: Thermochemical evolution of model S2: (a) Globally averaged internal temperature, (b) average heat flux across surface $\left(q_{s}\right)$ and CMB $\left(q_{c}\right)$, (c) Total mass (cumulative) of erupted material $M_{e}^{t o t}$ normalised by the total mantle mass $M=\int_{V_{m}} \rho d V$, where $V_{m}$ is the volume of the modelled spherical shell. Time is typically given in Ga and thus denotes time before present-day. For reference, the time since the start of the model (in Gyr) is indicated on top of panel (a). (d-e) Laterally averaged radial profiles of temperature and composition at different times, respectively. In (d) the dotted line denotes the solidus assuming the initially homogeneous composition shown as a dotted line in (e).

exist in this model. Also, the compositional profile does not seem to change much over the last $2-3 \mathrm{Gyr}$ of evolution, which may indicate that crustal production and recycling are in approximate equilibrium.

The quantitative details of the processes discussed in this section vary to some degree between the different models of the S-family, like the magnitude of surface and CMB heat flux, the timing of the thermal maximum and the total amount of erupted material generated in the course of the evolution. Qualitatively, the discussed behaviour seems typical for the stagnant-lid models. Some related diagnostics are listed in Table 3, but we note that no direct observations are available on the temperature within the venusian mantle or the heat flux across the surface.

\subsubsection{Gravity spectra and gravity-topography relations}

The model-predicted present-day gravity power spectra and their relation to topography are presented in Figure 2. A first general observation is that none of our generic stagnant-lid models resembles the observed gravity power spectrum closely over the whole range of spherical harmonic degrees $l=2-16$, but some cases perform significantly better than others (see diagnostics $\delta_{G}, \delta_{T}, \delta_{C}$ and $\delta_{C}$ in Table 3 ). Increasing the reference viscosity $\eta_{0}$ has a strong effect on the predicted gravity spectra by generally increasing the power and by shifting the peak 
power to somewhat higher spherical harmonic degrees (Figure 2b). Greater viscosity generally enhances convective stresses, which ultimately increases surface topography in our approach, so that increased gravity power may be somewhat expected. The shift of the peak power towards higher harmonics (or shorter wavelength) is less intuitive. Upon cooling with time, however, the mantle flow pattern typically evolves towards longer wavelength. This happens in all our stagnant-lid cases, but the process is slower with higher viscosity and cases S3 and S4 may thus still be dominated by too short flow components to explain Venus' presently observed topography and gravity spectra. This is also reflected in the detected number of mantle plumes (see Figure 3 in section 3.1.3). In contrast, cases S1 and S2 match the power spectrum significantly better.

All four models (S1-S4) feature a high correlation between gravity and topography as observed on Venus, but case S1 features somewhat reduced correlation in the range $l=5-8$ for unknown reasons (Figure 2c). The spectral ratio between gravity and topography $R_{g t}$ is matched very well by case $\mathrm{S} 2$, although this may exclude the longest wavelength $(l=2$, Figure $2 \mathrm{~d})$. At least for spherical harmonic degrees $l \leq 10$, case S2 seems to match the characteristics of Venus' present-day gravity and topography spectra best (see Table 3 ), although the fit is clearly not optimal $\left(\delta_{G}=0.52, \delta_{T}=0.40, \delta_{C}=0.48, \delta_{R}=0.25\right)$.

Next, we keep $\eta_{0}$ fixed at the value used in case S2, but vary the viscosity increase with depth by changing the activation volume $V_{A}$ (Figure 2e). Neither reducing nor increasing the depth increase of viscosity helps to improve the match to the observed gravity spectrum though (Figure 2f) or the correlation to topography and the spectral ratio of the two properties (Figure $2 \mathrm{~g}+\mathrm{h}$ ). In fact, case S2 remains the best matching case. Thus far, we have only varied the depth gradient, which corresponds to a smooth increase of viscosity with depth. The viscosity increase may also include discontinuities across interfaces like the mantle phase transitions. On Earth, for instance, matching the geoid at the surface by dynamic flow models has typically required a significant viscosity jump across the $660 \mathrm{~km}$ phase boundary (e.g. Hager et al., 1985). When introducing a viscosity jump in our model, the most important consequence is a breakdown of gravity-topography correlation in the lower spherical harmonics that is the more pronounced the stronger the viscosity contrast across the $730 \mathrm{~km}$ phase transition is (Figure 2k). This is consistent with the lack of this correlation on Earth, but does not match the spectral characteristics of Venus as already suggested by previous studies (e.g. Steinberger et al., 2010; Huang et al., 2013).

Finally, changing the parameters $H_{P}$ and $\Delta H$ (see section 2) causes a slightly different thermal evolution. In both cases this leads to a somewhat reduced mantle temperature and correspondingly higher average mantle viscosity, but the effects seem rather small compared to those explained above (Figure $2 \mathrm{~m}$ ). The density anomalies defined by cases S2, S2a and S2b still differ, however, which may explain the difference in the predicted spectra (Figure 2n-p).

\subsubsection{Number of mantle plumes}

We now investigate the number of mantle plumes in the stagnant-lid evolutions (S1-S8), which has recently been used as a constraint on the venusian 

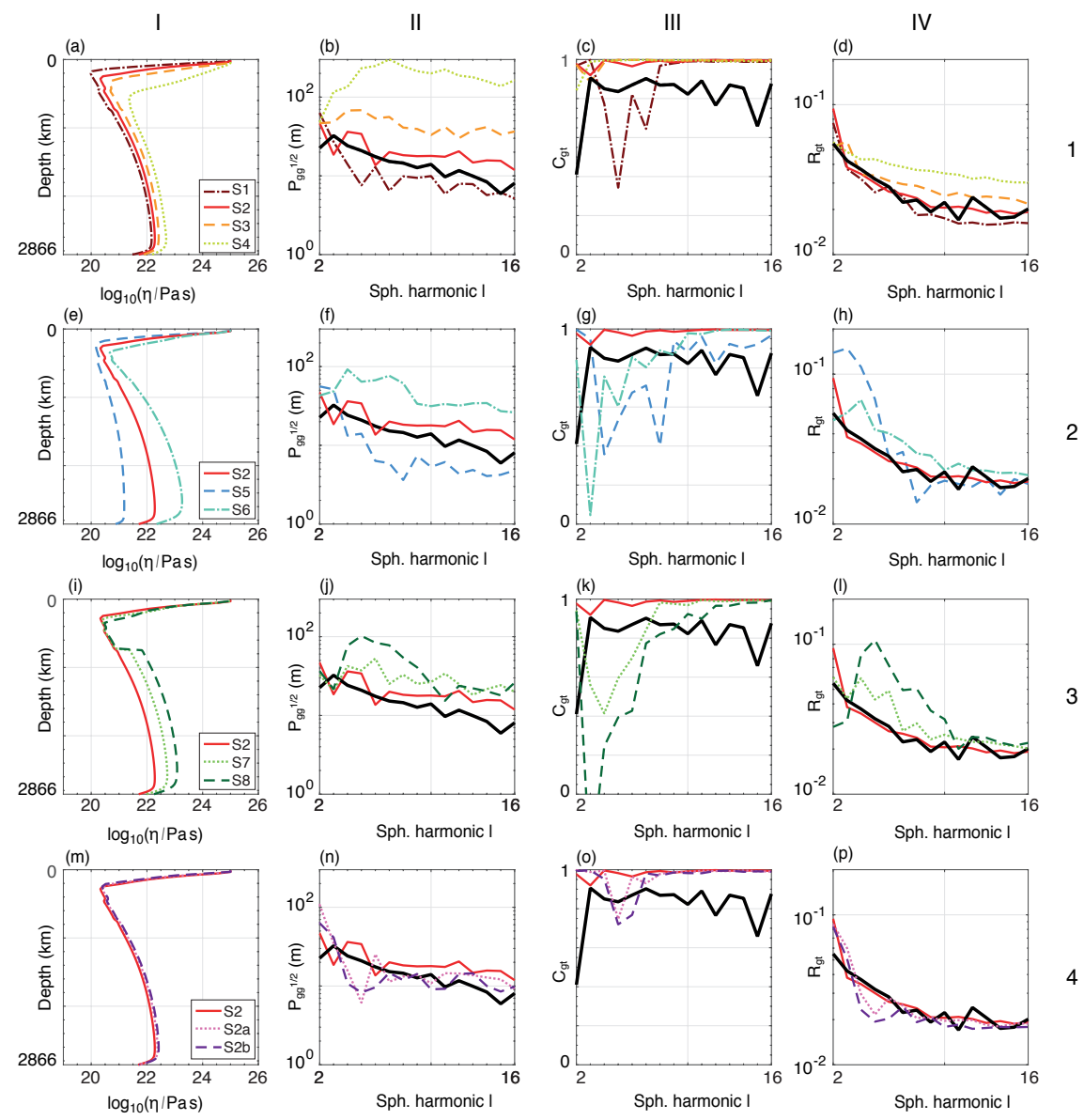

Figure 2: Gravity field spectral analysis for the stagnant-lid cases S1-S8 in comparison to observations for present-day Venus (thick black lines). Each row denotes a different family of cases in which a different parameter is varied: (Row 1) reference viscosity $\eta_{0}$, (Row 2) activation volume $V_{A 0}$, (Row 3 ) viscosity jump across the phase transition to perovskite. In (Row 4), a lower internal heating rate (parameter $H_{P}$, case S2a) or a stronger partitioning of radiogenic elements into the basaltic material (parameter $\Delta H$, case $\mathrm{S} 2 \mathrm{~b}$ ) has been employed. Each column I-IV displays a different measure: (I) present-day radial viscosity profiles; (II) the square-root of the present-day gravity power spectra $P_{g g}$ as defined in the text, (III) the degree correlation between gravity and topography $C_{g t}$ and (IV) the spectral ratio $R_{g t}$ of gravity and topography. 
volatile history (Smrekar and Sotin, 2012) and mantle viscosity structure (Huang et al., 2013). Mantle plumes may be linked to anomalies in Venus' surface thermal emissivity and only nine of them have been detected by the VIRTIS experiment on Venus Express (Smrekar et al., 2010). To accomplish this, we follow the methodology of Huang et al. (2013) and track hot mantle plumes based on their temperature and radial velocity characteristics. A hot plume is detected, when the local temperature $T_{l o c}$ at the depth of interest is significantly larger than the average $\left(T_{\text {avg }}\right)$ at this depth: $T_{l o c}>T_{a v g}+\xi_{1}\left(T_{\max }-T_{\text {avg }}\right)$, where $T_{\max }$ denotes the maximum temperature at this depth. The same criterion is used for radial velocity instead of temperature. For the plume regions detected by this method, we compute the plume flux and subsequently ignore all small plumes for which this flux is smaller than a fraction $\xi_{2}$ of the maximum flux. Following Huang et al. (2013), we chose $\xi_{1}=0.2$ and $\xi_{2}=0.05$, but we repeat the detection 100-times and let $\xi_{1,2}$ vary by up to $\pm 50 \%$ around these central values in order to evaluate the sensitivity of results with respect to our choice of $\xi_{1,2}$. If not stated otherwise, the number of plumes is analysed at a depth of $\sim 970 \mathrm{~km}$. We chose this rather deep detection layer because we are mostly interested in the major mantle plumes; towards shallower depth, the thermal structures are typically smaller-scale because of the lower viscosity and the action of magmatic processes, and it becomes more difficult to detect the relevant anomalies with our simple approach (Figure 3a-c).

We find that the uncertainty caused by the assumed $\xi_{1,2}$ is rather small in the stagnant-lid cases (except perhaps for the high-viscosity case S4): the computed standard deviation is typically $10 \%$ or less of the mean value (Table 3 and Figure 3d). Within the last billion years, the number of detected plumes does not seem to vary much in most cases, but during earlier phases of the evolution their number tends to be higher (by a factor of 1.5-2.5, Figure 3e). The initial plumes may then slowly merge to more pronounced groups in response to the long-term cooling of the mantle, which effectively decreases convective vigour. This process of plume merging seems to occur somewhat faster the less viscous the mantle is, which may explain why the number of computed mantle plumes for the present-day increases with the reference viscosity $\eta_{0}$ (cases S1-S4, Figure $3 \mathrm{~d}$ ). In fact, in the highest viscosity case (S4) the process of plume merging is probably far from being completed at $t=0 \mathrm{Ga}$.

A further, but less pronounced trend is that the number of detected plumes tends to increase with stronger viscosity stratification at depth. The trend seems to hold for both purely gradual and mixed gradual-discontinuous increases (compare cases S5-S2-S6 and S2-S7-S8 in Figure 3d, respectively). This would be in line with the argument in the previous paragraph since a stronger viscosity gradient would cause a more viscous lower mantle which probably controls the mobility of plume conduits that originate from the CMB.

The cases for which the spectral characteristics of gravity and topography were matched best (cases S1 and S2) also seem to comport with the VIRTIS constraint of approximately nine detected high thermal emissivity regions. This is not surprising since Venus' long-wavelength gravity spectrum is tied to the structure of mantle flow (Steinberger et al., 2010) of which the number and 

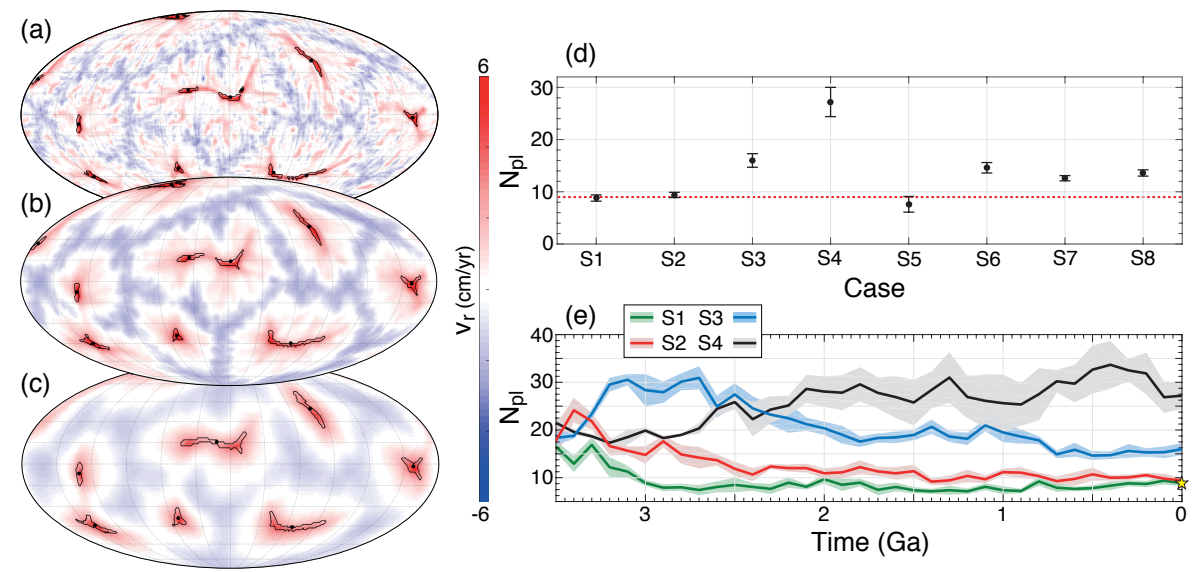

Figure 3: Mollweide projections of radial velocity $v_{r}$ (colour-coding) at depths of (a) $\sim 590 \mathrm{~km}$, (b) $\sim 940 \mathrm{~km}$, (c) $\sim 1850 \mathrm{~km}$ (case S2 at present-day); $v_{r}>0$ denotes upward motion. Black contours indicate detected plume regions whose centroids are depicted by black dots. Here, the detection parameters $\xi_{1}=0.2$ and $\xi_{2}=0.05$ are used (following Huang et al., 2013). (d) Number of detected plumes $N_{p l}$ at present-day for cases S1-S8. The dots indicate the mean values of 100 estimations with different $\xi_{1}, \xi_{2}$, which have been varied randomly by up to $\pm 50 \%$ from the values used in (a)-(c). The error bars depict the corresponding standard deviations. In (d), the dotted horizontal line is an estimate for present-day Venus using thermal emissivity constraints (Smrekar et al., 2010). (e) Time variation of $N_{p l}$ since $3.5 \mathrm{Ga}$ for cases S1-S4 in $100 \mathrm{Myr}$ increments. Bold lines indicate the mean number of plumes $\overline{N_{p l}}$, the shaded margins the standard deviation at the respective time step.

positioning of plumes is a representation. In contrast to the gravity spectrum, the number of plumes is an indirect observation since the proposed link between thermal emissivity anomalies and deep mantle plumes (see Smrekar et al., 2010) cannot be rigorously tested with existing available data. Accordingly, the number of plumes may thus not be as constraining for Venus evolution models as the gravity observation. Yet, our finding that reduced mantle viscosities are favourable for matching Venus' rather small number of plumes is generally consistent with the results of Smrekar and Sotin (2012), although these authors suggest the need of even lower mantle viscosity $\left(\leq 10^{20} \mathrm{Pas}\right)$ if strong internal heating is present. All our models are dominantly internally heated, but they also feature significant (gradual) viscosity variation below the lithosphere, which is known to affect the wavelength of convection. In this combination, which was not investigated by Smrekar and Sotin (2012), it seems possible to predict Venus' number of mantle plumes without the need of such very low mantle viscosities.

\subsection{Episodic evolutions}

\subsubsection{Thermal and magmatic evolution}

In the models described above, the lithosphere remained in a stagnant-lid state throughout the entire evolution, such that large-scale recycling of the 

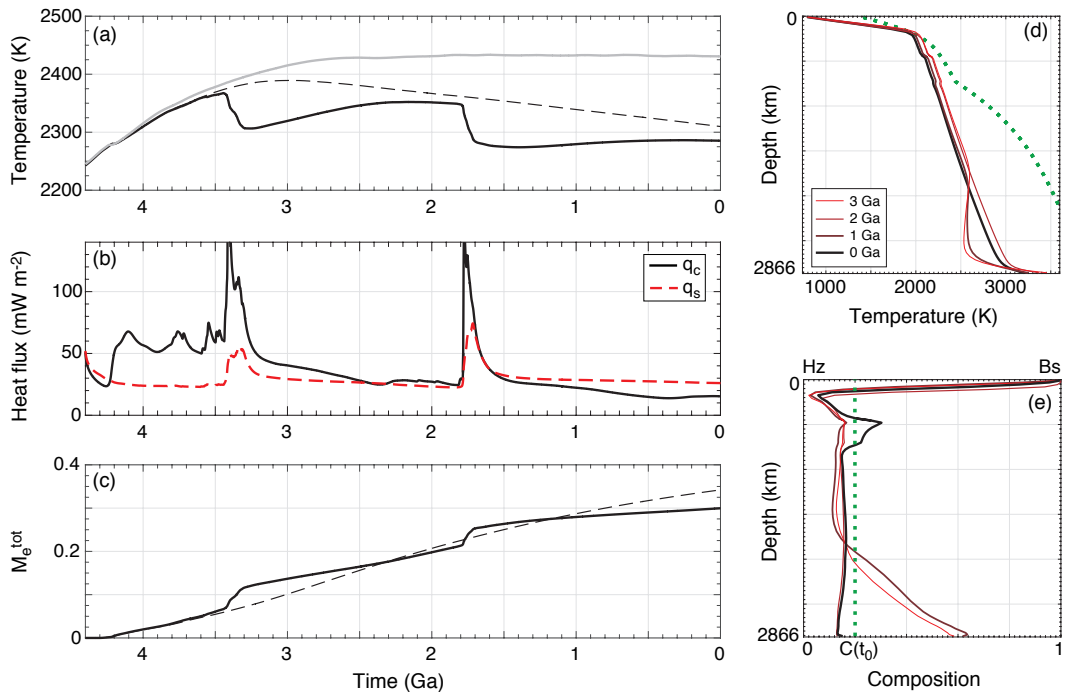

Figure 4: As in Figure 1, but for the episodic model E50. In (a) and (c), the results for the stagnant-lid evolution S2 are given for comparison as a dashed line. In (a), we also show the result for case E50 recomputed without the basalt-eclogite phase transition in grey.

surface by tectonic processes was inhibited. In this section, we present the other class of evolution models with phases of rapid surface mobilisation, which lead to tectonic recycling of parts or the entire surface. Here, we focus on episodic evolutions that have clearly distinguishable overturn events, separated by elongated phases of stagnant-lid convection since this allows us to investigate the overturn events and their aftermath in detail. For this purpose, we tune the yield stress of the lithosphere $\left(\sigma_{0}\right)$ to reach such a scenario, but note that the number, duration and frequency of such overturn events in Venus' history are unknown.

Case E50 features two major overturns the first of which initiates at $\sim 3.4 \mathrm{Ga}$, the second one at $\sim 1.8 \mathrm{Ga}$ (Figure 4 ). Upon recycling of cold surface material into the deeper interior, the mean temperature of the mantle drops (Figure 4a) and heat transport across the boundary layers becomes more efficient (Figure 4b). During the overturn, the total heat flux across the surface may be $2-3 \times$ higher than before the overturn. The heat flux increase is even stronger for the bottom heat flux once cold recycled material comes to rest on the CMB, which temporally increases the temperature drop across this boundary (Figure $4 \mathrm{~d}+\mathrm{e}$ ). In addition to the temporary accumulation of basaltic components at great mantle depth after the overturn event, this material can also become relatively enriched in the mantle transition zone as a consequence of the basalt barrier mechanism stating that basalt is not buoyant at this depth (Papuc and Davies, 2012). 


\subsubsection{Overturn evolution}

As in the stagnant-lid evolutions described above, melting and its extraction and thus magmatic surface recycling is ongoing throughout the entire evolution, but it peaks during the overturns and then happens at a clearly reduced rate after the overturn, also compared to the rate in the stagnant-lid evolution (Figure 4c). At present-day, however, the difference in total cumulated erupted material between cases S2 and E50 seems rather small ( 10-15\%, Table 3).

Generally, the overturn events in our model are typically triggered by the growth of crust. Upon ongoing melt extraction from the interior, the basaltic crust on the surface grows thicker. Once crustal thickness exceeds the eclogite phase transition at $\sim 65 \mathrm{~km}$ depth, the crust becomes more and more negatively buoyant and the resulting stresses in the lithosphere overcome the yield strength at some point. The importance of this process is additionally highlighted by the fact that recomputing the same model without the basalt-eclogite transition did not feature any overturn event (Figure 4a).

The overturn events may thus initiate locally according to the crustal and stress distribution. Once initiated the stress pattern induced to the lithosphere changes and lithospheric failure propagates rapidly across the surface (Figure $5)$. This process typically affects the surface globally, but we have not explicitly investigated here, whether parts of the surface may resist recycling during the overturn.

During the overturn, the surface is mobilised and may on average move as fast as $\sim 20 \mathrm{~cm} / \mathrm{yr}$. The duration of surface mobilisation is $\sim 150-200 \mathrm{Myr}$ (Figure 5e). In fact, this duration is very similar to what has been observed in corresponding 2D evolutions of Armann and Tackley (2012), which is somewhat surprising as one may expect a more complex propagation of resurfacing in $3 \mathrm{D}$ and thus a longer time required for global resurfacing. This may point to a rather symmetric style of overturn propagation that can be reasonably captured also by $2 \mathrm{D}$ models. The value of the yield stress $\left(\sigma_{0}\right)$ does not seem to affect this behaviour very much; the main consequence of changing $\sigma_{0}$ is a change in timing and perhaps the frequency of the overturns: with higher yield stress, the lithosphere can sustain the stress induced by mantle flow and crustal growth for a longer time.

It is interesting to note that the 2D models of Armann and Tackley (2012) predict $5-8$ overturns for a typical evolution of Venus. In contrast, our 3D models predict only $1-3$ overturns. Clearly, this depends on the details of the model setup and the resulting stresses in relation to the yield stress, although lowering its value in our models does not seem to lead to a significantly increased number of clearly distinguishable overturns. Instead, the system may fall into a state of (somewhat Earth-like) continuous recycling at some point (case E30 in Figure 5e, which has a $40 \%$ reduced yield stress compared to the reference model). This may point towards a different time-dependence in 2D and 3D models as was already suggested by Huang et al. (2013), although their models did not feature lithospheric overturn. 

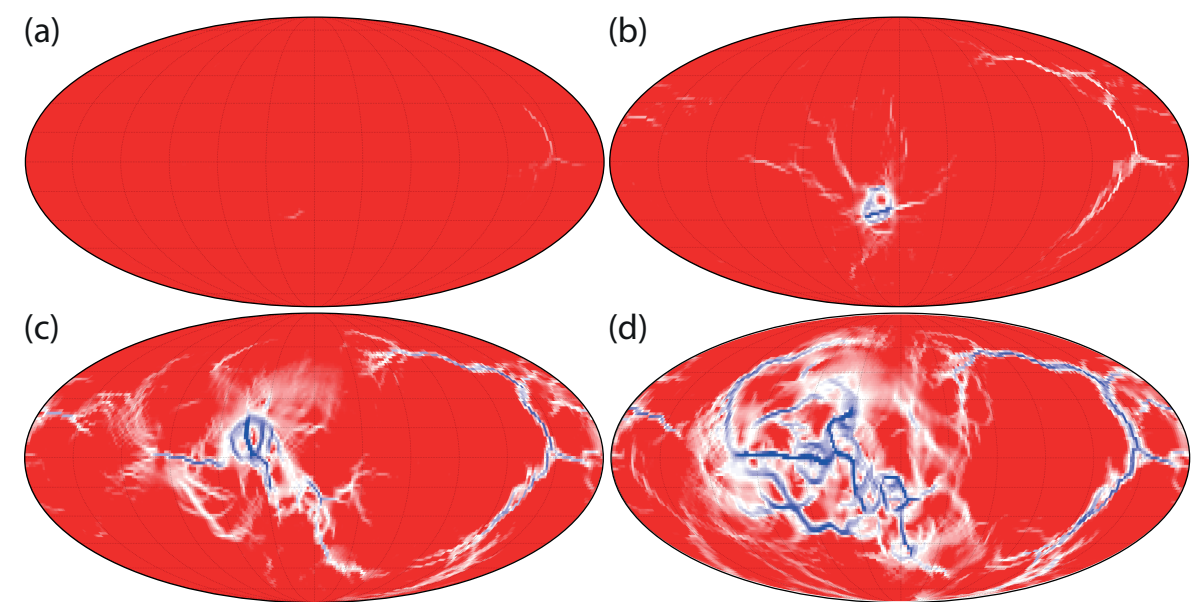

(d)

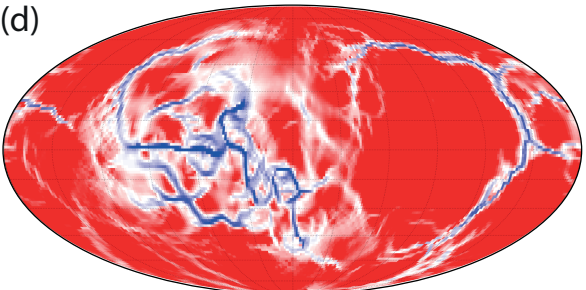

(e)

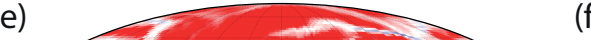

(f)
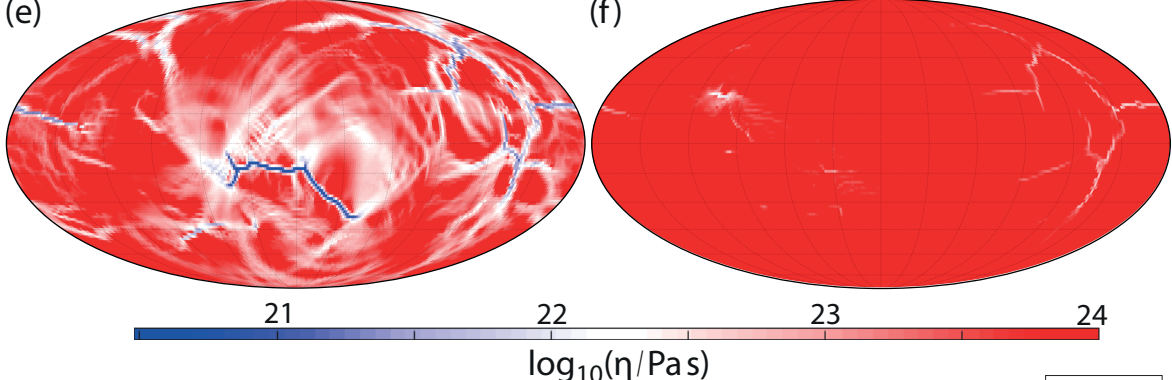

23 24

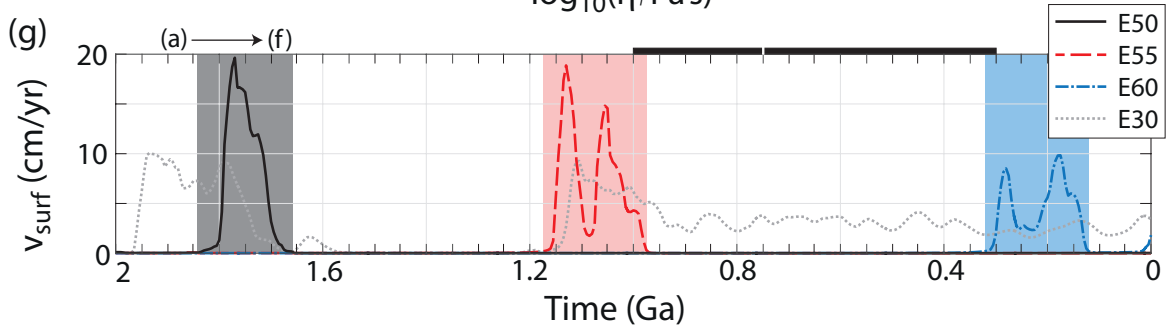

Figure 5: Overturn evolution (case E50). Mollweide projections of surface viscosity at different times: (a) $1.90 \mathrm{Ga}$, (b) $1.82 \mathrm{Ga}$, (c) $1.79 \mathrm{Ga}$, (d) $1.78 \mathrm{Ga}$, (e) $1.71 \mathrm{Ga}$, (f) $1.68 \mathrm{Ga}$. (g) Time evolution of rms-surface velocity (smoothed) since $2 \mathrm{Ga}$ for four cases with different surface yield stress $\sigma_{0}$ as indicated. The black solid curve corresponds to the evolution shown in (a)-(f). The shaded regions indicate the time period during which surface velocities are significantly increased due to the overturn. Note that Case E70 is not shown here, because it does not show significant surface mobilisation after $2 \mathrm{Ga}$, its last overturn fades around $2.4 \mathrm{Ga}$. For the other cases, the last overturn falls in the plotted time span. Venus' mean surface age (white mark) and its uncertainty range (black horizontal bar) as inferred from cratering statistics (McKinnon et al., 1997) are indicated on top of panel (g). 


\subsubsection{The aftermath of an overturn event}

As described in the previous section, the overturns can be seen as extreme events that globally perturb the background dynamics of the planet's interior. Thus, they introduce additional time scales into the thermal evolution, which are related to the frequency of overturn events and the time scale over which they may affect the planetary interior. Especially this latter time scale is of great interest for the interpretation of present-day planetary observations, such as gravity. As indicated above, overturns mobilise the surface globally and the duration of these mobilisation periods is estimated to be $150-200$ Myr based on our modelling. However, the recycled surface material may affect the state of the interior over a longer time period and this could be detectable in surface observables.

An analysis of the spectral characteristics of gravity and topography in some of our episodic cases is given in Figure 6. Again, several major observations can be made. First, with a too low yield stress that leads to an almost continuously overturning evolution (case E30), the cold recycled surface material leads to a stronger viscosity increase with depth (Figure 6a). As a consequence, the power spectrum of gravity decreases more strongly with increasing harmonic degree $l$ and results in a strongly $(l=2)$-dominated planet with comparably large misfit to the observed spectra (Table 3 and Figure 6b). In liaison, the correlation between gravity and topography in the low degree range breaks down as already observed in stagnant-lid models with strong viscosity increase with depth (Figure 6c). This clearly points to a more Earth-like rather than a Venus-like model. Similar effects, though somewhat less pronounced, can be observed for case E60 in which the latest overturn faded only very recently (Figure 5g) and regions of anomalously high viscosity in the lower mantle caused by cold recycled material still persist. On the other hand, some of the episodic cases in which the latest overturn event happens sufficiently long ago, generate an equally good or even better match to Venus' observed gravity spectrum than our most successful stagnant-lid model (S2). For example, case E50 predicts the smallest misfits in the gravity $\left.\delta_{g g}=0.26\right)$ and topography spectra $\left(\delta_{t t}=0.30\right)$ across our suite of cases. Perhaps the most remarkable difference is that the successful episodic cases also produce the observed peak in the gravity spectrum at spherical harmonic degree $l=3$ and the relatively lower power at $l=2$ (Figure $6 \mathrm{~b}$ ), which typically did not evolve in the stagnant-lid models (Figure 2). We note though that this $(l=3)$-dominance is only featured during a small part of the evolution since the last overturn event (Figure 6e), so the relevance of this observation is difficult to infer.

Clearly, the overturn event strongly perturbs the gravity power spectrum at all wavelengths. At the longest wavelengths $(l=2-3)$, the peak power during the overturn may be 1-2 orders of magnitude above the pre- and post-overturn level (Figure 6e), although the quantitative increase most likely depends on the details of our model. However, this peak is rather short and mostly coincides with the period of surface mobilisation. Some increased power in $l=2-3$ may still be visible after the surface motion has terminated, but is limited to 

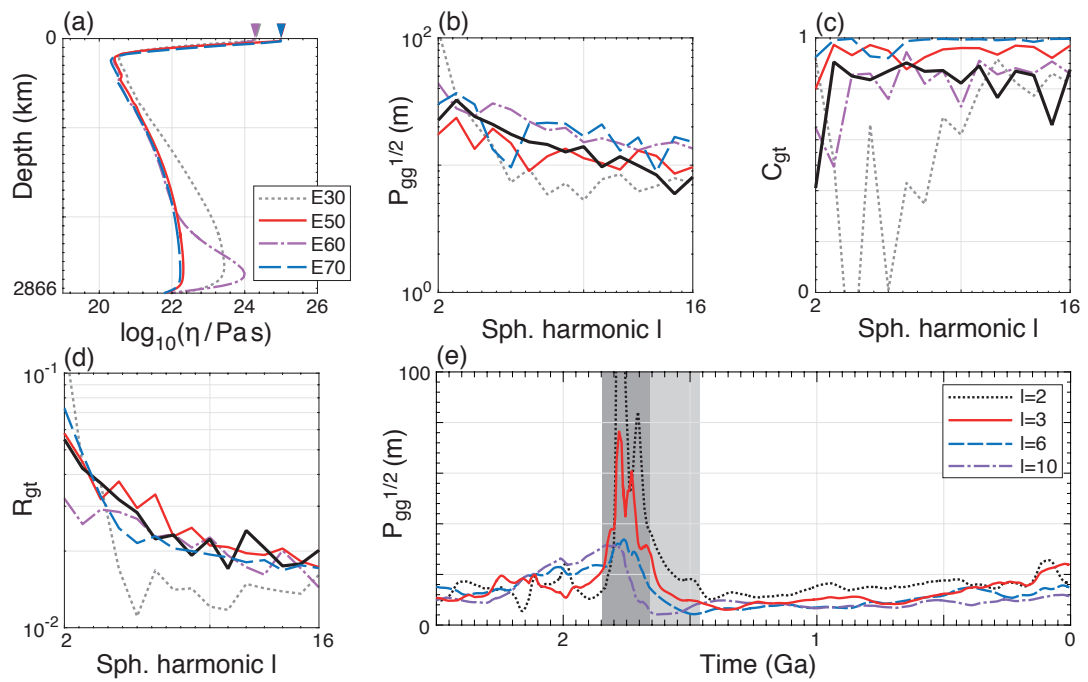

Figure 6: Spectral analysis for episodic cases: (a)-(d) correspond to panels (a)-(d) in Figure 2, but depict cases E30, E50, and E70 with different values of surface yield stress $\sigma_{0}$. (e) Time evolution of the power in spherical harmonic degrees $l=[2,3,6,10]$ since $2.5 \mathrm{Ga}$. The dark-shaded region indicates when the surface is substantially mobilised (compare to Figure 5 ), the light-shaded region indicates for how much longer the gravity power for $l=2-3$ still differs from the pre-overturn level.

$\sim 100-200 \mathrm{Myr}$. This is probably the case, because the cold surface material sinks rather rapidly through the mantle given our preferred viscosity profile without strong discontinuities. Such material will come to rest on top of the CMB (Figure 4e), but the surface gravity is rather insensitive to density anomalies in this lowermost depth range and certainly only at the longest wavelengths (e.g. Hager et al., 1985).

Such deeply recycled material on top of the CMB may also affect the CMB heat flux and the structure of the bottom boundary layer which in turn controls the initialisation of mantle plumes (Figure 7). Some time after the onset of the overturn, the recycled basaltic material will cover the major part of the CMB thereby annihilating the pre-overturn plume pattern. In the following, plumes have to initialise again, initially on small-scale. The number of plumes detected by our simple approach is very high then (Figure $7 \mathrm{~d}$ ), but their actual number is quite dependent on the detection parameters $\xi_{1,2}$. The key observation, however, is that their number recovers to approximately pre-overturn level once the recycled material has entrained into shallower mantle layers again (Figure 7e). In our models, this process requires a rather long time of $1 \mathrm{Gyr}$ or even more, but again, this will depend on the detailed density structure of the models and also the temperature at the CMB. Further systematic exploration of such parameters is necessary to further refine our general observation. If this holds true though and the number of mantle plumes is in addition indeed related to surface thermal emissivity anomalies, such a long overturn relaxation time could 

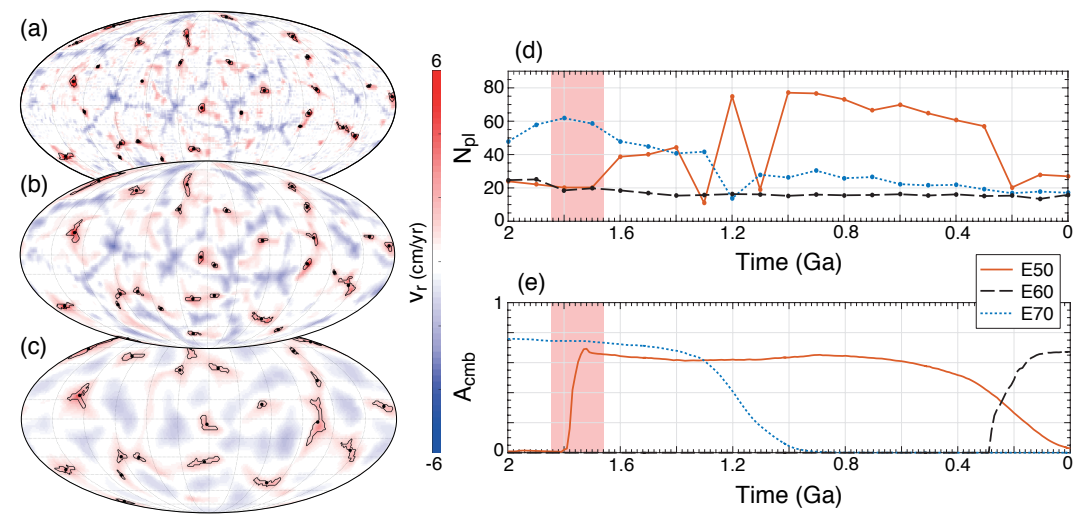

Figure 7: (a)-(c) As in Figure 3a-c, but for episodic case E50. (d) Time evolution of the mean number of detected plumes $\left(\overline{N_{p l}}\right)$ since $2 \mathrm{Ga}$ in episodic cases E50, E60 and E70. (e) Fraction of the CMB area $\left(A_{c m b}\right)$ covered by basaltic material. The shaded region indicates the period of major surface mobilisation for case E50.

favour a rather long-ago cessation time of Venus' latest overturn event. We note that it can also take some time after the overturn onset until the number of plumes increases substantially. Possibly, not all overturn events disturb the plume pattern equally strong (e.g., see evolution E60 since $\sim 0.4 \mathrm{Ga}$ in Figure $7 \mathrm{~d}$ ). This may depend on where the resurfacing event initiates and then how it propagates across the surface in relation to the plume pattern. Inferring these details is beyond the scope of the present study, but without them it is probably difficult to infer overturn cessation time from the plume pattern, respectively from the number of plumes.

\subsection{A stagnant or an episodic lid scenario for Venus?}

\subsubsection{Crustal thickness evolution}

So far, we have presented the differences between the stagnant-lid and episodic scenario, but it would be desirable to constrain which of these may be more applicable for Venus' evolution. One diagnostic to discuss is crustal thickness, which is essentially given by the local thickness of the surface layer of basaltic crust which has been extracted from the interior upon melting (see e.g. Keller and Tackley, 2009). Here, we are mostly interested in its spatial mean value and its standard deviation (see Table 3 for quantitative results).

Due to our initial condition, crustal thickness is initially zero in all our models until the onset of melting processes, that is after $\sim 100$ Myr. From then on mean crustal thickness increases for $1-2 \mathrm{Gyr}$ for the stagnant-lid models until a maximum is reached (Figure 8a), afterwards mean crustal thickness appears rather constant indicating a balance between production of new crust due to melt extraction and destruction of crust by convective erosion and drip-off of the dense eclogitic base of the crust (see also Armann and Tackley, 2012). The slight decrease of crustal thickness towards modern times as observed in 
some cases is probably an expression of secular cooling due to which magmatism slowly fades (Figure 1).

In none of the stagnant-lid cases a present-day mean crustal thickness of less than $100 \mathrm{~km}$ can be observed, which is significantly above previous independent estimates (e.g. Anderson and Smrekar, 2006; James et al., 2013; Wei et al., 2014). This may mean that too much melting is generated in our model (or at least erupted onto the surface). We tried to reduce the amount of melting by allowing for enrichment of radiogenic heat sources in the basaltic component (case S2b), so that they should concentrate in the crust in the course of the evolution. Yet, this seems to only marginally reduce crustal thickness, similar to the findings of Armann and Tackley (2012) in 2D models. Even reducing the (present-day) bulk internal heating rate by $20 \%$ does not reduce present-day mean crustal thickness greatly, but mostly effect the timing of crustal growth (case S2a in Figure 8a).

In the episodic models much reduced crustal thickness can be achieved, again mostly depending on the timing of the last overturn event (Figure 8b). In the stagnant-lid phases of these evolutions, crustal thickness grows according to the rate of melt extraction. Too thick eclogitic crust, however, triggers an overturn to reset crustal thickness. It still remains difficult to generate really small average crustal thicknesses, probably because overturn events also feature substantial magmatism and new crust will already be emplaced somewhere, while recycling is on-going elsewhere. Consistent with Armann and Tackley (2012), we note that the episodic cases can still feature some eclogitic crustal base to some extent, probably because the crust is embedded in a thicker lithosphere which prevents efficient basal recycling of the crust.

Nevertheless, several of our episodic evolutions generate present-day mean crustal thicknesses that reasonably overlap with other estimates. As in the stagnant-lid models, we tested also the effect of reduced bulk internal heating and abundance of radiogenic elements in the crust (cases E50a and E50b). Both tend to reduce the effective growth rate of crust in the stagnant-lid phases of the evolutions, however, the time that has passed since the last overturn seems to be the most important controlling parameter.

\subsubsection{Mean surface age}

Another important constraint on Venus' evolution comes from its impact crater population, which cannot be distinguished from a random distribution (Herrick, 1994). This and the relatively small number of craters (less than 1000) has lead to the view that Venus' surface has a spatially rather uniform mean age of $0.75_{-0.45}^{+0.25}$ Gyr (McKinnon et al., 1997), although the degree of uniformity and its spatial scales is an issue of on-going research (e.g. Kreslavsky et al., 2015, also see discussion in section 4.2). This relatively young age implies substantial resurfacing during Venus' evolution. While an evolution with catastrophic overturns seems more feasible to achieve the observed characteristics (e.g Romeo and Turcotte, 2010), it may also be possible to achieve these via equilibrium resurfacing, for example via volcanic activity (e.g. Bjonnes et al., 2012).

Our numerical models allow us to compile global age distributions at any 

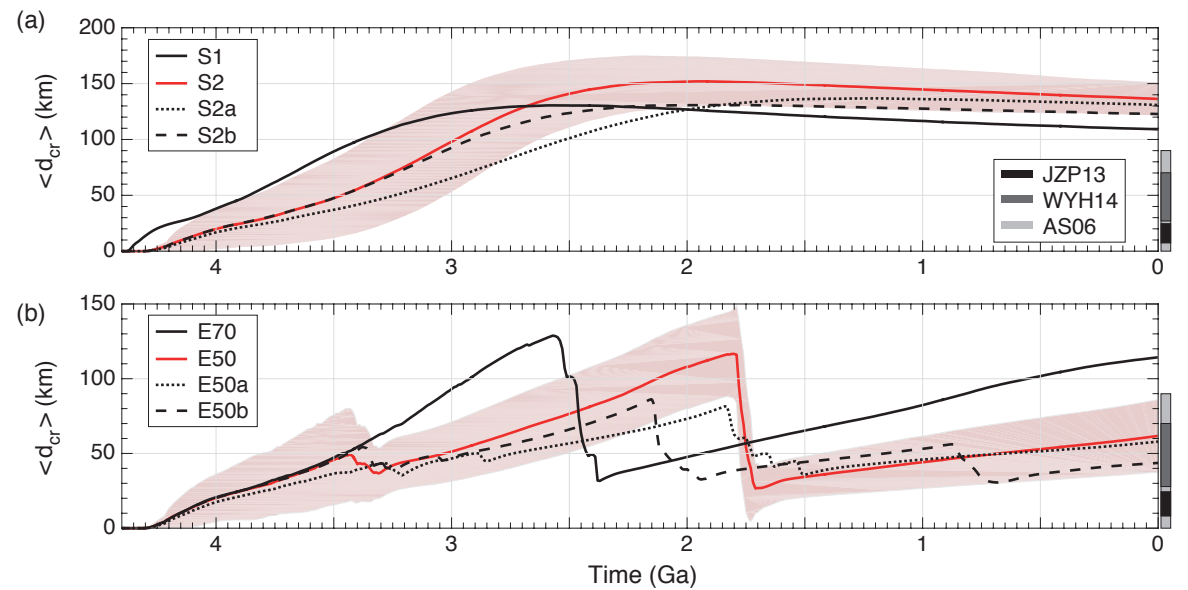

Figure 8: Evolution of modelled mean crustal thickness $\left\langle d_{c r}\right\rangle$ for (a) stagnant-lid cases and (b) episodic-lid cases as indicated in the legend. Lines depict the computed mean ages, the shaded areas indicate the standard deviation for cases S2 and E50, respectively. The grey bars on the right $\mathrm{y}$-axis denote independent estimates from the literature (JZP13: James et al., 2013), (WYH2014: Wei et al., 2014), (AS06: Anderson and Smrekar, 2006).

given time of the evolution and thus provide important insight into the origin of Venus' present surface age spectrum. We extract surface age from our models by tracking the time a tracer particle has spent in the topmost cell of the numerical grid and averaging the age over all tracer particles within each surface grid cell. This method captures recycling via both magmatism and lithospheric overturn the latter of which has been ignored in previous efforts to analyse Venus' surface age with convection models (Noack et al., 2012). But our simple approach has also limitations and typically leads to somewhat noisy surface ages that can vary strongly over short length scales. The mean age $\bar{A}$, however, seems to be a rather robust estimate independent of these small-scale fluctuations.

As a typical stagnant-lid evolution, case S2 features a mean surface age of $\bar{A} \sim 0.25 \pm 0.18$ Gyr. This mean value does not seem to vary strongly (less than a factor of 2) within 4 Gyr of model evolution (Figure 9). Reducing the amount of melting and thus the efficiency of magmatic surface recycling by reducing the bulk internal heating rate (case S2a) and by increasing the abundance of heatproducing elements in the basaltic crust (case S2b) helps to increase the mean age slightly, but not to more than $0.30-0.35$ Gyr. In contrast, the episodic model E50 features substantially larger mean age $(\bar{A} \sim 0.60 \pm 0.40 \mathrm{Gyr})$ for the present venusian surface to which it has evolved from the latest overturn that happened at $\sim 1.8 \mathrm{Ga}$. During the overturn, surface age is reset to almost zero as expected.

We note that the predicted present-day mean age is significantly less than the time passed since the latest overturn, which indicates the strong role of magmatic resurfacing in our models. This may also explain why the surface age 


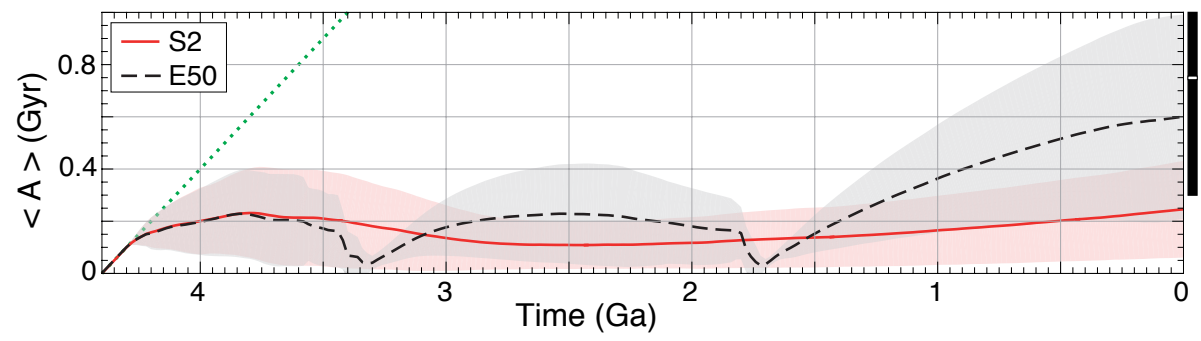

Figure 9: Time evolution of mean surface age $\langle A>$ for (a) case $\mathrm{S} 2$, (b) case E50. The shaded areas indicate the respective standard deviation. The vertical bar on the right indicates the proposed range of Venus' present mean surface age (e.g. McKinnon et al., 1997). The dotted line indicates the maximum possible age (i.e., the complete absence of resurfacing).

distribution appears far from uniform as indicated by the rather strong lateral variation (Figure 9) as ongoing volcanism will degrade the age distribution after the overturn. As indicated by the rather larger standard deviations as given above ( $\sim 75 \%$ and $\sim 66 \%$ with respect to the respective mean age), none of our models is currently able to meet the uniformity constraint of Venus' present-day surface in a strict sense. More research is required to understand which conditions are feasible to achieve age distribution with both reasonable mean age and small lateral variation in our model (see section 4.3), but we also deem it necessary to further evaluate the degree of age uniformity on current Venus and over which length scales it may apply.

\section{Discussion}

\subsection{Venus' mantle viscosity structure and its implications}

Based on the ability of our models to match present-day surface observations, we deem case E50 as most representative for Venus' evolution at least in its later stages following the last overturn event. This case predicts a minimum viscosity of $\sim 2 \times 10^{20} \mathrm{Pas}$ in Venus' shallow sublithospheric mantle at $\sim 200 \mathrm{~km}$ depth. This depth of minimum viscosity seems consistent with the lithospheric thickness estimate at $\sim 200 \mathrm{~km}$ of Benesova and Cizkova (2012), who also used a 3D spherical convection model to infer mantle viscosity from gravity observations, but did not consider thermochemical effects, melting and magmatism. On the other hand, Orth and Solomatov (2011) suggested a lithospheric thickness of up to $600 \mathrm{~km}$ based on the assumption that Venus long-wavelength topography is mostly explained by isostatically compensated variations in stagnant-lid thickness. From the shallow minimum, viscosity then increases gradually with depth by a factor of $\sim 100$ to a depth of $\sim 2600 \mathrm{~km}$ (or $\sim 250 \mathrm{~km}$ above the CMB) in our model.

This preferred viscosity profile is almost identical to the one inferred by Steinberger et al. (2010), based on mineral physics constraints (Steinberger and Calderwood, 2006). In contrast to their work, which tended to overpredict 
Venus' gravity power spectrum at longest wavelength, $l=2-4$, our thermochemical forward modelling approach is also capable of reasonably matching the power spectrum and its relation to topography at these spatial scales (Figure $6)$. This includes the absence of a $(l=2)$-dominance, at least intermittently, which is one of the striking differences between the gravity spectra of Venus and Earth (and in fact also of Mars, Mercury and the Moon).

Steinberger et al. (2010) based their findings on geoid and topography kernels, which do not consider lateral variations in viscosity, which are present in our approach. However, lateral variations have probably a minor effect on the surface gravity and topography at the longest wavelength compared to radial variations (Richards and Hager, 1989). In addition, the magnitude of lateral viscosity variations in the absence of subducting slabs or large-scale chemical heterogeneity is probably small anyway within Venus' deep interior compared to Earth's. Steinberger et al. (2010) assumed that mantle density heterogeneity has the same spectral characteristic on present Earth and Venus instead of computing the evolution of density heterogeneities forward in time as we have done here. This may explain parts of the difference in predicted gravity power at long-wavelength between Steinberger et al. (2010) and our model. By assuming the same spectral and depth dependence of density anomalies for both planets, Steinberger et al. (2010) implicitly considered the decrease of thermal expansivity with depth. Reduced thermal expansivity in the deep mantle could reduce the magnitude of density anomalies there, which would relatively reduce the power in the lowest degrees of the gravity spectrum (only the longest wavelengths are sensitive to the deep mantle). On the other hand, lower expansivity would reduce convective vigour in the lower mantle, which could give rise to a less time-dependent and longer-wavelength flow pattern that reinforces the power in the lower degrees. Which of these effects may dominate is undetermined at this stage and requires further modelling in future. Finally, we note that all models of Steinberger et al. (2010) assumed a stagnant-lid scenario in which our models also tend to overpredict the spectrum at least at $l=2$ (Figure 2), perhaps because this essentially implies infinite material strength which could alter the stress patterns in the lithosphere.

On the other hand, several studies have recently demonstrated that dynamic forward modelling of venusian mantle convection in the stagnant-lid regime has the ability to predict the long-wavelength power spectrum closely (e.g. Benesova and Cizkova, 2012; Huang et al., 2013). While these studies also consider selfconsistent thermal evolution of the venusian mantle in $3 \mathrm{D}$, even with lateral viscosity variations (Huang et al., 2013), they did not consider an evolutionary framework as we have done here, including secular cooling, compositional variation, melting and overturn events. Interestingly, Huang et al. (2013) inferred an almost flat viscosity profile below the lithosphere with a total viscosity increase of a factor of $\sim 5$ towards the CMB, which is lower or on the very low end of what has been found in our and other previous studies (e.g. Pauer et al., 2006; Benesova and Cizkova, 2012). Huang et al. (2013) highlight the importance of phase transitions and their properties in this matter, which have been neglected by various studies (e.g. Benesova and Cizkova, 2012). However, they required a 
large Clapeyron slope for the transition to perovskite $\left(\gamma_{o l_{730}}=-3.5 \mathrm{MPaK}^{-1}\right)$. This is larger than the value we have used here $\left(\gamma_{o l_{730}}=-2.0 \mathrm{MPaK}^{-1}\right)$. This value also seems rather large compared to inferences from recent experiments (e.g. Kojitani et al., 2016), unless the mantle at the transition pressure is sufficiently hydrous, which may increase the absolute value of $\gamma$ to levels comparable to their choice (Ghosh et al., 2013). On the other hand, such a hydrous mantle may not be expected for Venus (e.g. Grinspoon, 1993).

If such details of the phase transitions explain why Huang et al. (2013) were able to use a much smaller viscosity increase with depth to match Venus' observed gravity spectrum, then more future effort should indeed be spent to improve the treatment of mineral physics in mantle convection models in order to capture their impact on mantle dynamics sufficiently well. Already, Armann and Tackley (2012) reported that mineral phase transitions increased the timedependence in their 2D models, although this has not been observed in the (simpler) 3D models of Huang et al. (2013). We have not varied phase transition parameters here, so cannot assess this question directly. Still, our models support the importance of phase transitions in the sense that the basalt-eclogite transitions seems to be the dominant trigger for overturn events (Figure 4), although this transition is only relevant at shallow rather than mantle transition zone depth.

In line with some previous work on Venus' mantle viscosity structure as cited in the previous paragraphs, our models confirm that no significant viscosity discontinuity across the transition zone should exist, because it seems at odds with the high correlation between geoid and topography as inferred for Venus. This points to important differences in the internal mantle structure of Earth and Venus, since for the Earth a viscosity jump across the transition zone is typically necessary to fit the surface geoid observation (e.g. Hager et al., 1985). Possibly, the structural difference between the two bodies can be explained by a hydrous terrestrial upper mantle and transition zone, perhaps due to subduction-triggered water cycling, and a relatively dry upper mantle in Venus due to the absence of such a process. Venus' interior may have been dried out additionally by an early large impact (Davies, 2008). In fact, the on-going magmatism in the upper mantle suggested by our models (Figures 1c and 4c) would lead to outgassing of volatiles and water and a rather dry upper mantle (e.g. Grinspoon, 1993; Smrekar and Sotin, 2012), although probably not entirely dry (Elkins-Tanton et al., 2007). If the lower mantle is comparably dry as Earth's (e.g. Murakami et al., 2002), this could explain a relatively smooth viscosity increase with depth in the venusian mantle that is governed by pressure and temperature changes, but not by water content, which may in contrast be relevant for Earth. This remains somewhat speculative, however without coupling our interior evolution model to water and volatile content and their effects on effective viscosity. Several recent models of Venus coupling interior and atmosphere consider transport of water and volatiles from the mantle into the atmosphere upon melt extraction (e.g. Noack et al., 2012; Gillmann and Tackley, 2014). Yet, none of them considers the direct effects of water on viscosity though (cf. Richard and Bercovici, 2009) with which it could ultimately be 
tested whether the more gradual venusian mantle viscosity profile is explained by a different water distribution than in the terrestrial mantle.

\subsection{The evolutionary state of Venus' present interior}

In all our models volcanic resurfacing is ongoing at present-day as previously reported by the numerical studies of Armann and Tackley (2012) and Gillmann and Tackley (2014). Ongoing very recent resurfacing also seems to be in line with current observational inferences from Venus' surface (Smrekar et al., 2010; Bjonnes et al., 2012). The style of resurfacing on modern Venus may also be via localised plume-triggered subduction as observed in recent laboratory-scale experiments and some of the venusian coronae (Davaille et al., 2017). While our episodic cases do feature some local deformation in the shallow lithosphere also in the stagnant-lid phases between overturns, the stresses are too small to induce such localised subduction and/or coronae formation. This may require a more complex rheology that may include distinct crustal and mantle rheologies and additional mechanisms for crustal and lithospheric weakening (e.g. Gerya, 2014).

With these limitations aside, resurfacing in our models away from large-scale overturn events happens via melt extraction (magmatic/volcanic recycling). Stagnant-lid models then feature large mean crustal thicknesses of $>100 \mathrm{~km}$ well beyond the basalt-eclogite transition depth. As already inferred by Armann and Tackley (2012) in 2D models this is not substantially improved when heat-producing elements are more abundant in the basaltic crust (Figure 8) and similarly if Venus' bulk internal heating rate was moderately lower for some reason. Such thicknesses are well beyond other independent estimates that mostly come from spectral admittance modelling. For instance, Anderson and Smrekar (2006)suggested a global range of crustal thickness from $0-90 \mathrm{~km}$ and Steinberger et al. (2010) derived a mean value of $\sim 60 \mathrm{~km}$ based on matching Venus' gravity power-spectrum at $l>40$, which is likely dominated by crustal contributions. More recently, Wei et al. (2014) used the convection model solutions of Huang et al. (2013) to correct for the dynamic contributions that affect long-wavelength topography and geoid and inferred a smaller range of crustal thicknesses on Venus of $28-70 \mathrm{~km}$ and James et al. (2013) even suggested a mean crustal thickness of Venus of only $8-25 \mathrm{~km}$. Crustal thickness estimates from spectral admittance modelling are intrinsically non-unique (e.g. Wieczorek, 2007), but all these studies consistently predict thinner crust than inferred from our stagnant-lid models (Table 3 ). With an upper mantle viscosity about a factor $\sim 30$ lower than inferred from our best fit model, average crustal thickness may be lower (Armann and Tackley, 2012) and closer to these observational estimates. Indeed, we observe the same trend (case S2 vs. S1), but constraints on numerical resolution currently do not allow us to reduce upper mantle viscosity further in our 3D model. However, we also note that the fit to observed spectral characteristics of gravity and topography starts to degrade for our lowest viscosity case (S1, see Figure 2a-d), so crustal thickness may become more realistic, but the predicted gravity power spectra may not.

In line with Armann and Tackley (2012), episodic overturns are the most 
feasible way to realise crustal thicknesses as inferred above. In contrast to the stagnant-lid models, crustal thickness is self-regulated in episodic models since too thick crust will trigger an overturn and thus reset crustal thickness, potentially globally. This way, our models can match above inferences at least at their upper end. We note, that mean crustal thickness still tends to be rather high, which may be linked to the simplifications in the melt extraction model (see section 4.3).

Regardless of the absolute value, the growth of crustal thickness between major overturn events seems to occur almost linearly (Figure 8b). For instance, in our preferred model E50, the crust thickens with a rate of $\sim 18 \mathrm{~km} /$ Gyr since the last overturn. Unfortunately, this rate seems to vary even for the same concentration of radiogenic elements in the mantle depending on previous evolution and thus the yield strength (e.g., compare cases E50 and E70 in Figure $8 \mathrm{~b}$ ), which is largely unconstrained. Moreover, mean crustal thickness is not reset to zero after the overturn, but to a finite value that typically lies between $25-35 \mathrm{~km}$ in our set of evolutions. Consequently, inferring the possible cessation time of the last venusian overturn event from present crustal thickness estimates and (linear) back-interpolation of crustal growth rates seems inappropriate at this point.

A more obvious way of inferring overturn cessation time would be by using the age distribution of Venus' surface. In our stagnant-lid models the (upper) mantle stays very hot throughout the entire evolution, which enhances melting and magmatism and thus leads to stronger rates of volcanic resurfacing: this leads to present-day mean surface ages of $<350 \mathrm{Myr}$ (Figure 9). In contrast, the episodic evolutions tend to feature clearly reduced volcanic eruption rates (e.g., Figure 4c). It is thus easier to maintain an older surface, so that mean surface ages of $\sim 600 \mathrm{Myr}$ can be generated. Such values are more in line with constraints from the cratering population (McKinnon et al., 1997), although the possibility of a very young surface for Venus has recently been suggested by Bottke et al. (2016), who revisited venusian impactor fluxes. The episodic models thus outperform the stagnant-lid models in terms of mean surface age (as for the gravity spectra and crustal thicknesses), so that this diagnostic supports the occurrence of an overturn in the venusian evolution. Stagnant-lid evolutions, on the other hand, seem more feasible in generating a uniform surface age in an absolute sense (see Figure 9). When lateral age variations are put into relation with the respective mean age, however, this observation can vanish and even turn around, that is the episodic evolution can even predict slightly better uniformity than the stagnant-lid evolution. Nonetheless, our current model does not succeed in predicting uniform age distributions in a strict sense, independent of the evolution scenario.

As a consequence, we put the emphasis on mean age rather than uniformity for this study, also because the degree of uniformity remains under debate for the case of present Venus whose surface age is probably also not strictly uniform (e.g. Nikishin, 1990; Basilevsky and Head, 2002). For example, tesserae terrains may be as old as $1.47 \pm 0.46$-times the mean age (Ivanov and Basilevsky, 1993) while some lava flow fields and large volcanoes may be as young as $0.41 \pm 0.29$ - 
and $0.23 \pm 0.15$-times the mean age, respectively (Price and Suppe, 1994). Recently, Kreslavsky et al. (2015) suggested that the average age of young (old) units may be $\sim 0.4(1.2)$-times the mean surface age. A detailed comparison of model-predicted surface age distributions with geological constraints needs to be performed in future. This may provide further insight into resurfacing rates and/or the time passed since the latest overturn of Venus' lithosphere.

That our current models have difficulty in generating uniform age distributions could be due to spatially heterogeneous magmatic activity as it happens more frequently in hotter regions, for instance the locations of hot upwellings. An uniform surface age would require that volcanic resurfacing is either shut down or happens everywhere at a comparable rate. While our stagnant-lid cases tend to the latter, this scenario seems unlikely for present-day Venus. The strong degradation of the uniform age distribution in our modelling may also be rooted in our simple melting model in which all magmatism is extrusive. In reality, most magmatism will be intrusive and will thus not directly contribute to resurfacing (see section 4.3).

At this stage, it remains difficult to decide whether the stagnant-lid or the episodic models generate a more Venus-like age distribution. With on-going magmatism, it can only be said that the presently observed mean surface age is a minimum estimate for the time passed since the latest overturn event: the more volcanic resurfacing has happened after the overturn ceased, the larger is the time difference $\Delta t$ between cessation time and mean surface age. In our best-fit model (E50), $\Delta t$ is large ( $\sim 1 \mathrm{Gyr})$, but this is probably an overestimation because we ignore volcanic intrusions as explained above. Nevertheless, a large $\Delta t$ is in line with our observation that the pattern of mantle plumes requires a long time to recover from the latest overturn and form a pattern that is characteristic of the stagnant-lid scenario (Figures 3 and 7 ). The small number of observed surface thermal emissivity anomalies (Smrekar et al., 2010) suggests that the plume pattern has readjusted after the overturn already and is representative of the stagnant-lid phase of the evolution. We cannot ultimately exclude the possibility that the plume pattern has not (yet) responded substantially to a rather recent overturn event (e.g., see case E60, Figure 7d), but this would probably imply a very young surface in conflict with most age estimates of the venusian surface. Clearly, more future work is required to refine the time scale of plume recovery and how it depends on the properties of the lower mantle including the bottom thermal boundary layer. Also, it is not well established that each mantle plume causes a thermal emissivity anomaly or whether anomalies may also be triggered by different processes.

The surface gravity spectrum is certainly a more robust constraint than the observed plume pattern, but according to our models it cannot help to further constrain the cessation time of the last overturn since the remnants of largescale surface recycling vanish quickly after the end of the overturn $(\sim 150 \mathrm{Myr}$, Figure 6e). Thus, the present-day spectrum should be clearly representative of a stagnant-lid phase, unless recycled material is somehow kept more efficiently at shallower depth ranges that influence surface gravity more than the CMB region where recycled material accumulates in our models. Armann and Tackley 
(2012) predicted a somewhat stronger accumulation of basaltic material in the transition zone as we observed here, probably due to details in the density structure of the transition zone (density jumps and Clapeyron slopes of the different phase transitions, depth-dependent thermodynamic parameters and compressibility). This may promote density anomalies in this region of the mantle, but the results of Armann and Tackley (2012) (see their Figure 5) suggest that this effect decreases with increasing reference viscosity $\eta_{0}$, so that the effect should be rather small in our presumably most-representative case of Venus (E50).

As a summarising note from our modelling, it seems that model-predicted gravity and topography as well as crustal thickness and mean surface age constraints are in better agreement with observational inferences when an episodic lid regime with a least one catastrophic overturn is considered. However, the slow recovery of the plume pattern after an overturn may suggest that the current stagnant-lid phase on Venus is ongoing for quite some time. A new overturn event may occur in future.

\subsection{Limitations}

We have presented self-consistent models of Venus' thermochemical mantle evolution in full 3D spherical geometry including global episodic overturns. This is an advancement from previous attempts to model Venus' interior. Our approach still has limitations several of which have been discussed above already, for example neglecting mantle compressibility and the depth-dependence of thermodynamical parameters, which may alter the density structure of the mantle. We also assumed the absence of water and volatile cycling and we have not varied the initial condition of our model, although this may not have great influence given the long time scale of evolution and the vigorously convecting mantle.

No interaction between Venus' atmosphere and the interior has been assumed, but recently, Gillmann and Tackley (2014) have shown that mantle outgassing can change the composition of the atmosphere, which may lead to changes in Venus' surface temperature and may ultimately trigger overturn events. This may alter the frequency, style, and duration of overturns and ultimately the thermomagmatic evolution of the whole planet (see e.g. Foley and Driscoll, 2016). In a further step, Gillmann et al. (2016) demonstrated that this coupled system may also be affected by asteroidal impacts, which erode the atmosphere and could be another trigger for overturn events.

The modulation of surface temperature by interior-atmosphere coupling may also impact damage and healing processes in the lithosphere, which could be important for the initialisation of surface mobilisation and the persistence of weak zones (e.g. Bercovici and Ricard, 2014). This possibly affects Venus' evolution particularly if its surface temperature variations are large: Gillmann and Tackley (2014) present temporal fluctuations of several 100s of K, thus comparable to the difference between Venus and Earth. More generally, our triggering mechanism for lithospheric overturns, that is when convective stresses overcome a yield stress, is simplified. This is indicated by the essentially unconstrained value of the yield stress, which is largely a tuning parameter. Other mechanisms 
such as grain size evolution (e.g. Foley and Bercovici, 2014) could be important and may change the style of surface recycling.

But perhaps the most important simplification in our model is the treatment of magmatic processes. In particular, we assume that all melt above a critical depth will trigger extrusive volcanism. However, this is not true for the Earth and probably for other planetary bodies including Venus, where the majority is intrusive, although the exact ratio is debated. Intrusive magmatism has recently been shown to strongly affect crustal and lithospheric dynamics on early-Earth (Rozel et al., 2017) and it is likely that hot intrusions may also modify the thermomechanical state of the venusian lithosphere and thus the planet's resurfacing mode (Tackley et al., 2014). These aspects should be considered in future work.

\section{Conclusions}

We have investigated the thermal, compositional, magmatic and tectonic evolution of the planet Venus using the mantle convection code StagYY in realistic 3D spherical geometry. Our main goal has been to infer Venus present-day state, in particular its mantle viscosity structure and to infer the evolutionary path of Venus based on present-day observables. Our results may be synthesised in the following concluding remarks:

1. In our stagnant-lid evolution models, Venus' observed power spectrum of surface gravity and its relation to topography is best-matched when sublithospheric mantle viscosity at $\sim 200 \mathrm{~km}$ depth is $\sim 2 \times 10^{20} \mathrm{Pas}$ and increases gradually with depth by a factor of $\sim 100$ to a maximum value of $\sim 2 \times 10^{22} \mathrm{Pas}$ at around $250-300 \mathrm{~km}$ above the core-mantle boundary. A stronger viscosity increase, particularly if caused by a discontinuity in the transition zone, is unfavourable as it inhibits the strong correlation of gravity and topography observed for Venus. The lack of such a viscosity increase in the transition zone on Venus may point to different water contents in the upper mantles of Venus and Earth, where the latter is more hydrated and thus features lower viscosity. Our most-representative stagnant-lid models generate a plume pattern in line with thermal emissivity constraints of Venus' surface, but always lead to too thick basaltic crust $(>100 \mathrm{~km})$ and tend to feature too young surface age $(<300 \mathrm{Myr})$.

2. Evolutions with a few episodic overturns generate very similar viscosity structures as in the stagnant-lid mode if the last overturn event has ceased for a sufficiently long time. In these models, the spectral characteristics of Venus' gravity and topography can be matched even better than in the stagnant-lid models, in particular at the longest wavelength. Such evolutions predict a much reduced crustal thickness $(\sim 40-60 \mathrm{~km})$ in much better consistency with previous estimates and more reasonable mean surface age $(\sim 600 \mathrm{Myr})$, but on the other hand a more complex evolution of the mantle plume pattern that may need a long time to recover from an overturn event. 
3. Overturn events may mobilise the surface globally for $\sim 150-200 \mathrm{Myr}$ and may perturb the predicted gravity power spectra for up to another $\sim 150-$ $200 \mathrm{Myr}$ after surface mobilisation as ceased. This may provide a minimum estimate of the cessation of Venus' latest global overturn event and suggest that the present venusian mantle should not contain any remnants of this overturn, perhaps with the exception of the region atop the core mantle boundary where overturn remnants may reside for much longer time and perturb the development of a stable plume pattern comparable to the stagnant-lid evolutions.

If our model observations from 1.-3. hold true, our work favours a venusian evolution that is currently in the stagnant-lid regime, but has featured at least one global event of tectonic recycling, which may have ceased a rather long time ago. Clearly, more observational data from Venus will be necessary in the future to confirm our suggestions.

\section{Acknowledgements}

All authors receive funding from the Norwegian Research Council through a Centre of Excellence grant to the Centre for Earth Evolution and Dynamics (CEED, 223272). Further support has been received through CRATER CLOCK (235058/F20). All computations have been performed on Stallo, a Notur highperformance computing facility at the University of Troms $\varnothing$, under project codes nn9283 and nn9010. The authors gratefully thank Paul J. Tackley for providing his StagYY code, Nicola Tosi and Stephen Mojzsis for two constructive reviews, and the editor Oded Aharonson for handling the manuscript.

\section{References}

Anderson, F., Smrekar, S., 2006. Global mapping of crustal and lithospheric thickness on Venus. J. Geophys. Res. 111, E08006.

Armann, M., Tackley, P., 2012. Simulating the thermochemical magmatic and tectonic evolution of Venus's mantle and lithosphere: Two-dimensional models. Jour. Geophys. Res. 117, E12003.

Basilevsky, A., Head, J., 2002. Venus: Timing and rates of geologic activity. Geology 30, 1015-1018.

Benesova, N., Cizkova, H., 2012. Geoid and topography of Venus in various thermal convection models. Stud. Geophys. Geod. 56, 621-629.

Bercovici, D., 2003. The generation of plate tectonics from mantle convection. Earth Planet. Sci. Lett. 205, 107-121.

Bercovici, D., Ricard, Y., 2014. Plate tectonics, damage and inheritance. Nature 508, 513-518. 
Bjonnes, E., Hansen, V., James, B., Swenson, J., 2012. Equilibrium resurfaing of Venus: Results from new Monte Carlo modeling and implications for Venus surface histories. Icarus 217, 451-461.

Bottke, W., Vokrouhlicky, D., Ghent, B., Mazrouei, S., Robbins, S., Marchi, S., 2016. On Asteroid Impacts, Crater Scalings Laws, and a Proposed Younger Surface Age for Venus. Lunar Planet. Sci. Conf. XLVII, \#2016.

Christensen, U., Yuen, D., 1985. Layered Convection Induced by Phase Transitions. J. Geophys. Res. 90, 10291-10300.

Davaille, A., Smrekar, S.E., Tomlinson, S., 2017. Experimental and observational evidence for plume-induced subduction on Venus. Nat. Geosci. 10, 349-355.

Davies, J., 2008. Did a mega-collision dry Venus' interior? Earth Planet. Sci. Lett. 268, 376-383.

Driscoll, P., Bercovici, D., 2013. Divergent evolution of Earth and Venus: Influence of degassing, tectonics, and magnetic fields. Icarus 226, 1447-1464.

Elkins-Tanton, L., Smrekar, S., Hess, P., Parmentier, E., 2007. Volcanism and volatile recycling on a one-plate planet: Applications to Venus. J. Geophys. Res. 112, E04S06.

Foley, B., Driscoll, P., 2016. Whole planet coupling between climate, mantle, and core: Implications for rocky planet evolution. Geochem. Geophys. Geosyst. $17,1885-1914$.

Foley, B.J., Bercovici, D., 2014. Scaling laws for convection with temperaturedependent viscosity and grain-damage. Geophys. J. Int. 199, 580-603.

Gerya, T., 2014. Plume-induced crustal convection: 3D thermomechanical model and implications for the origin of novae and coronae on Venus. Earth Planet. Sci. Lett. 391, 183-192.

Ghosh, S., Ohtani, E., Litasov, K., Suzuki, A., Dobson, D., Funakoshi, K., 2013. Effect of water in depleted mantle on post-spinel transition and implication for $660 \mathrm{~km}$ seismic discontinuity. Earth and Planetary Science Letters 371, $103-111$.

Gillmann, C., Golabek, G., Tackley, P., 2016. Effect of a single large impact on the coupled atmosphere-interior evolution of Venus. Icarus 268, 295-312.

Gillmann, C., Tackley, P., 2014. Atmosphere/mantle coupling and feedbacks on Venus. Jour. Geophys. Res. 119, 1189-1217.

Grinspoon, D.H., 1993. Implications of the high D/H ratio for the sources of water in Venus' atmosphere. Nature 363, 428-431. 
Hager, B., Clayton, R., Richards, M., Comer, R., Dziewonski, A., 1985. Lower mantle heterogeneity, dynamic topography and the geoid. Nature 313, 541545 .

Herrick, R., 1994. Resurfacing history of Venus. Geology 22, 703-706.

Herzberg, C., Condie, K., Korenaga, J., 2010. Thermal history of the Earth and its petrological expression. Earth Planet. Sci. Lett. 292, 79-88.

Huang, J., Yang, A., Zhong, S., 2013. Constraints of the topography, gravity and volcanism on Venusian mantle dynamics and generation of plate tectonics. Earth Planet. Sci. Lett. 362, 207-214.

Ivanov, M., Basilevsky, A., 1993. Density and morphology of impact craters on tessera terrain, Venus. Geophys. Res. Lett. 20, 2579-2582.

James, P., Zuber, M., Phillips, R., 2013. Crustal thickness and support of topography on Venus. J. Geophys. Res. 118, 859-875.

Keller, T., Tackley, P., 2009. Towards self-consistent modeling of the martian dichotomy: The influence of one-ridge convection on crustal thickness distribution. Icarus 202, 429-443.

Kojitani, H., Inoue, T., Akaogi, M., 2016. Precise measurements of enthalpy of postspinel transition in $\mathrm{Mg} 2 \mathrm{SiO} 4$ and application to the phase boundary calculation. J. Geophys. Res. 121, 729-742. 2015JB012211.

Kreslavsky, M., Ivanov, M., Head, J., 2015. The resurfacing history of Venus: Constraints from buffered crater densities. Icarus 250, 438-450.

McKinnon, W., Zahnle, K., Ivanov, B., Melosh, H., 1997. Venus II. University of Arizona Press, Tucson, Azizona. chapter Cratering on Venus: Models and observations. pp. 969-1014.

Moresi, L., Solomatov, V., 1998. Mantle convection with a brittle lithosphere: thoughts on the global tectonic styles of the Earth and Venus. Geophys. J. Int. 133, 669-682.

Murakami, M., Hirose, K., Yurimoto, H., Nakashima, S., Takafuji, N., 2002. Water in Earth's Lower Mantle. Science 295, 1885-1887.

Nikishin, A., 1990. Tectonics of venus: A review. Earth Moon Planets 50/51, 101-125.

Noack, L., Breuer, D., Spohn, T., 2012. Coupling the atmosphere with interior dynamics: Implications for the resurfacing of Venus. Icarus 217, 484-498.

O'Neill, C., Marchi, S., Zhang, S., Bottke, W., 2017. Impact-driven subduction on the Hadean Earth. Nature Geosci. 10, 793-796. 
Orth, C., Solomatov, V., 2011. The isostatic stagnant lid approximation and global variations in the Venusian lithosphere. Geochem. Geophys. Geosyst. 12, Q07018.

Papuc, A., Davies, G., 2012. Transient mantle layering and the episodic behaviour of Venus due to the 'basalt barrier' mechanism. Icarus 217, 499-509.

Pauer, M., Fleming, K., Cadek, O., 2006. Modeling the dynamic component of the geoid and topography of Venus. J. Geophys. Res. 111, E11012.

Price, M., Suppe, J., 1994. Mean age of rifting and volcanism on Venus deduced from impact crater densities. Nature 372, 756-759.

Richard, G., Bercovici, D., 2009. Water-induced convection in the Earth's mantle transition zone. J. Geophys. Res. 114, B01205.

Richards, M., Hager, B., 1989. Effects of Lateral Viscosity Variations on LongWavelength Geoid Anomalies and Topography. J. Geophys. Res. 94, 1029910313.

Romeo, I., Turcotte, D., 2010. Resurfacing on Venus. Plan. Space Sci. 58, $1374-1380$.

Rozel, A., Golabek, G., Jain, C., Tackley, P., Gerya, T., 2017. Continental crust formation on early Earth controlled by intrusive magmatism. Nature 545,332 .

Schubert, G., Sandwell, D., 1995. A Global Survey of Possible Subduction Sites on Venus. Icarus 117, 173-196.

Smrekar, S., Sotin, C., 2012. Constraints on mantle plumes on Venus: Implications for volatile history. Icarus $217,510-523$.

Smrekar, S., Stofan, E., Mueller, N., Treiman, A., Elkins-Tanton, E., Helbert, J., Piccioni, G., Drossart, P., 2010. Recent Hotspot Volcanism on Venus from VIRTIS Emissivity Data. Science 605, 605-608.

Solomatov, V., 1995. Scaling of temperature- and stress-dependent viscosity convection. Phys. Fluids 7, 266-274.

Steinberger, B., Calderwood, A., 2006. Models of large-scale viscous flow in the Earth's mantle with constraints from mineral physics and surface observations. Geophys. J. Int. 167, 1461-1481.

Steinberger, B., Holme, R., 2002. An explanation for the shape of Earth's gravity spectrum based on viscous mantle flow models. Geophys. Res. Lett.eo $29,2019$.

Steinberger, B., Werner, S., Torsvik, T., 2010. Deep versus shallow origin of gravity anomalies, topography and volcanism on Earth, Venus and Mars. Icarus 207, 564-577. 
Steinberger, B., Zhao, D., Werner, S., 2015. Interior structure of the Moon: Constraints from seismic tomography, gravity and topography. Phys. Earth Planet. Int. 245, 26-39.

Tackley, P., 2008. Modelling compressible mantle convection with large viscosity contrasts in a three-dimensional spherical shell using the yin-yang grid. Phys. Earth Planet. Int. 171, 7-18.

Tackley, P., Ammann, M., Brodholt, J., Dobson, D., Valencia, D., 2013. Mantle dynamics in super-Earths: Post-perovskite rheology and self-regulation of viscosity. Icarus $225,50-61$.

Tackley, P., King, S., 2003. Testing the tracer ratio method for modeling active compositional fields in mantle convection simulations. Geochem. Geophys. Geosyst. 4, 2001GC00214.

Tackley, P., Lourenco, D., Golabek, G., 2014. Influence of intrusive magmatism on Venus' tectonics and long-term thermo-chemical mantle evolution. European Planet. Sci. Congress 9, 532.

Turcotte, D., Schubert, G., 2002. Geodynamics. Cambridge University Press, Cambridge. 2nd edition.

Turcotte, D., Willemann, R., Haxby, W., Norberry, J., 1981. Role of Membrane Stresses in the Support of Planetary Topography. J. Geophys. Res. 86, 39513959 .

Wei, D., Yang, A., Huang, J., 2014. The gravity field and crustal thickness of Venus. Science China: Earth Sciences 57, 2025-2035.

Weller, M., Lenardic, A., O'Neill, C., 2015. The effects of internal heating and large scale climate variations on tectonic bi-stability in terrestrial planets. Earth Planet. Sci. Lett. 420, 85-94.

Wieczorek, M., 2007. Treatise on Geophysics. Elsevier. volume Volume 10: Planets and Moons. chapter Gravity and Topography of the Terrestrial Planets. 2 edition.

Xie, S., Tackley, P., 2004. Evolution of U-Pb and Sm-Nd systems in numerical models of mantle convection and plate tectonics. J. Geophys. Res. 109, B11204.

Zhang, S., Christensen, U., 1993. Some effects of lateral viscosity variations on geoid and surface velocities induced by density anomalies in the mantle. Geophys. J. Int. 114, 531-547.

Zhong, S., McNamara, A., Tan, E., Moresi, L., Gurnis, M., 2008. A benchmark study on mantle convection in a 3-D spherical shell using CitcomS. Geochem. Geophys. Geosyst. 9, Q10017. 